ARTICLE

\title{
Short antisense oligonucleotides alleviate the pleiotropic toxicity of RNA harboring expanded CGG repeats
}

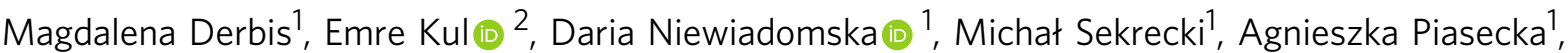 \\ Katarzyna Taylor ${ }^{1}$, Renate K. Hukema (i) ${ }^{3,4}$, Oliver Stork ${ }^{2}$ \& Krzysztof Sobczak ${ }^{1 凶}$
}

Fragile X-associated tremor/ataxia syndrome (FXTAS) is an incurable neurodegenerative disorder caused by expansion of CGG repeats in the FMR1 5'UTR. The RNA containing expanded CGG repeats (rCGGexp) causes cell damage by interaction with complementary DNA, forming R-loop structures, sequestration of nuclear proteins involved in RNA metabolism and initiation of translation of polyglycine-containing protein (FMRpolyG), which forms nuclear insoluble inclusions. Here we show the therapeutic potential of short antisense oligonucleotide steric blockers (ASOs) targeting directly the rCGGexp. In nuclei of FXTAS cells ASOs affect R-loop formation and correct miRNA biogenesis and alternative splicing, indicating that nuclear proteins are released from toxic sequestration. In cytoplasm, ASOs significantly decrease the biosynthesis and accumulation of FMRpolyG. Delivery of ASO into a brain of FXTAS mouse model reduces formation of inclusions, improves motor behavior and corrects gene expression profile with marginal signs of toxicity after a few weeks from a treatment.

\footnotetext{
${ }^{1}$ Department of Gene Expression, Institute of Molecular Biology and Biotechnology, Adam Mickiewicz University, Uniwersytetu Poznanskiego 6, Poznan, Poland. ${ }^{2}$ Department of Genetics and Molecular Neurobiology, Institute of Biology, Otto von Guericke University, Magdeburg, Germany. ${ }^{3}$ Department of Clinical Genetics, Erasmus MC, CA Rotterdam, The Netherlands. ${ }^{4}$ Present address: Department of Health Care Studies, Rotterdam University of Applied Sciences, HR Rotterdam, The Netherlands.凶email: ksobczak@amu.edu.pl
} 
S everal genetic disorders caused by the expansion of trinucleotide CGG repeats (CGG ${ }^{\exp }$ ) in the $5^{\prime}$ untranslated region (5'UTR) of the FMR1 gene located on chromosome $\mathrm{X}$ have been described. The most severe is fragile X syndrome (FXS), which is the most common cause of intellectual disability and autism in men. FXS results from large expansions containing over 200 CGG repeats, which cause complete silencing of the FMR1 gene and, therefore, a lack of fragile $\mathrm{X}$ mental retardation protein (FMRP), which plays an important role in synaptic plasticity ${ }^{1}$. Milder expansions with 55-200 CGG repeats lead to the development of either fragile X-associated primary ovarian insufficiency (FXPOI), which manifests as premature menopause, or a late-onset neurodegenerative disorder called fragile X-associated tremor/ataxia syndrome (FXTAS) ${ }^{2-4}$. Due to incomplete penetrance of the mutation, approximately 1 in 5000 men older than 50 suffer from FXTAS ${ }^{4}$. Random inactivation of chromosome X in women greatly reduces the risk of disease development ${ }^{4}$. The main clinical features of FXTAS include intention tremor, gait ataxia, and cognitive decline. Neuropathy, mild brain atrophy, and white matter lesions develop progressively in the presence of ubiquitinpositive intranuclear inclusions in neurons and astrocytes accompanied by a twofold to eightfold increase in the FMR1 mRNA level ${ }^{2-5}$.

The molecular basis of FXTAS is still incompletely understood, but three main pathomechanisms of this disorder have been proposed (Fig. 1a). First, as the 5'UTR region of FMR1 is GC-rich, the cotranscriptional formation of R-loop structures can be induced $^{6-8}$. These RNA/DNA hybrids formed by newly synthesized RNAs containing expanded CGG repeats (rCGG ${ }^{\exp }$ ) and complementary DNA strands containing CCG repeats may induce local DNA damage causing cell death ${ }^{3,6,8}$. R-loop formation depends on the CGG repeat length, hence, CGG ${ }^{\exp }$ hairpins stabilize this structure substantially ${ }^{8}$. Second, mutated FMR1 mRNA can interact with many nuclear RNA-binding proteins (RBPs) and form inclusions in the nucleus of neurons in the brain of patients with FXTAS ${ }^{9-16}$. This mechanism is known as RNA toxicity. The sequestration of proteins such as DGCR8 (DiGeorge Syndrome Critical Region 8) and SAM68 (Src-associated in mitosis $68 \mathrm{KDa}$ protein) interferes with miRNA maturation and the alternative splicing of SAM68-dependent genes, respectively ${ }^{13,15}$. Third, repeat-associated non-AUG (RAN) translation of CGG $^{\exp }$ can yield toxic polyglycine-containing proteins (FMRpolyGs) that are prone to form nuclear inclusions in FXTAS patients' brains ${ }^{17-19}$. The sequence of the $5^{\prime} \mathrm{UTR}$ of FMR1 carrying rCGG ${ }^{\exp }$ is translated beginning at near-cognate ACG or GUG start codons upstream of the CGG repeats. In addition, the natural product of this gene, FMRP, is produced starting at a canonical start codon downstream of the CGG repeats within a different reading frame ${ }^{18,19}$.

The three proposed pathomechanisms have one molecule in common, rCGG ${ }^{\text {exp}}$, suggesting that FXTAS could be highly amenable to RNA targeting interventions; however, no curative therapy is currently available. A few reports have suggested that small compounds targeting $\mathrm{CGG}^{\exp }$ within FMR1 mRNA have therapeutic potential. In an FXTAS cell model, some RNA-binding compounds reduced SAM68-dependent aberrant splicing and inhibited RAN translation of FMRpolyG ${ }^{20-22}$. In a similar cell model, we showed that antisense oligonucleotides (ASOs) complementary to the FMR1-specific sequence downstream of the CGG repeat tract induced targeted mRNA degradation through RNase $\mathrm{H} 1$ recruitment and caused a significant decrease in FMRpolyG production and aggregation ${ }^{23}$. Recently, ASOs targeting RAN translation initiation site on mRNA was also proved to reduce biosynthesis of FMRpolyG in FXTAS cellular models ${ }^{24}$. However, none of these compounds have been tested in vivo.
The aim of this work was to determine the therapeutic potential of short chemically modified ASOs directly targeting rCGG $^{\exp }$ in FMR1 mRNA. We used ASO steric blockers that bind to complementary sequences without inducing mRNA degradation and reduce the overall toxic effect of expanded repeats. Here, we present evidence that short ASOs complementary to rCGG ${ }^{\exp }$ (ASO-CCG) can alleviate not only molecular hallmarks of FXTAS, such as cotranscriptional R-loop formation, nuclear rCGG ${ }^{\exp }$-mediated toxicity, and FMRpolyG biosynthesis but also FXTAS-related molecular and behavioral phenotypes after long-lasting infusion of ASO-CCG directly into a brain ventricle in a mouse model of FXTAS.

\section{Results}

ASO-CCG binds rCGG $^{\text {exp }}$ with high affinity and releases nuclear proteins from sequestration. Pure CGG repeat tracts in RNA (rCGGs) form the most stable hairpin structure of all trinucleotide RNA repeats (e.g., rCUG, rCAG, and rCCG), with a melting temperature of $\sim 80^{\circ} \mathrm{C}^{25}$. Moreover, rCGG hairpins are further stabilized by flanking sequences within FMR1 mRNA ${ }^{26}$ and are thus not readily available to interact with other nucleic acids. Therefore, ASOs designed to bind to rCGG repeats should be characterized by a higher affinity and thermodynamic stability for ASO/RNA duplexes formed in trans than for RNA/RNA hairpins formed in cis. Thus, we designed ASO blockers with locked nucleic acid-modified phosphorothioate oligonucleotides (LNA-PSs) and CCG repeat sequences (i.e., ASO-CCG). One oligomer has 9 LNA residues; the other, 11. As a control, we used ASO-ctrl with a random sequence, with the same chemical modifications and corresponding lengths (either 9 or $11 \mathrm{nt}$ ).

As a first step, we sought to determine whether different types of ASO-CCG can efficiently invade the rCGG hairpin in vitro. We performed an electrophoretic mobility shift assay (EMSA) in which different concentrations of two ASO-CCGs were used to calculate their affinity for radioactively labeled $\mathrm{rCGG}_{20}$. Both ASO-CCGs exhibited high affinity, especially the shorter ASO variant, with a dissociation constant $\left(K_{d}\right)$ in the high picomolar range (Fig. $1 \mathrm{~b}$ and Supplementary Fig. S1a). Next, we sought to determine whether ASO-CCGs can efficiently enter living cells. Based on our previous study ${ }^{27}$ testing ASOs of different sequences but composed exclusively of LNA units, we anticipated that $\sim 20-30 \%$ of ASO-CCG may enter treated cells. Fluorescently labeled ASO-CCGs delivered via lipofection passed through the cell membrane, producing a unique dispersed signal in cells (Supplementary Fig. S1b). Moreover, the results of fluorescence microscopy suggested that almost all cells received comparable amounts of ASO-CCG. We checked also the general toxicity of tested ASOs in comparison to an ASO showing significant sequence-related toxicity used as a positive control (toxic ASO). During the time-course experiment, the viability of cells treated with ASO-CCG and ASO-ctrl was similar to mock-treated cells (Fig. 1c). In addition, a flow cytometry assay revealed that short LNA-based ASOs did not contribute to apoptosis (Fig. 1d). Therefore, we assumed that both control and rCGG repeattargeting oligonucleotides analyzed in this study show no or very low cell toxicity.

The high affinity and bioavailability, as well as low cellular toxicity of ASO-CCGs, encouraged us to examine their effects on (1) RNA toxicity, (2) RAN translation efficiency, and (3) cotranscriptional R-loop formation in different FXTAS cell models. First, we tested whether ASO-CCGs treatment could rescue the activity of the nuclear proteins DGCR8 and SAM68, involved in miRNA processing and RNA splicing, respectively, which are sequestered by the hairpin structures formed by rCGG ${ }^{\exp }$. We used artificial FXTAS cell models in which CGG 


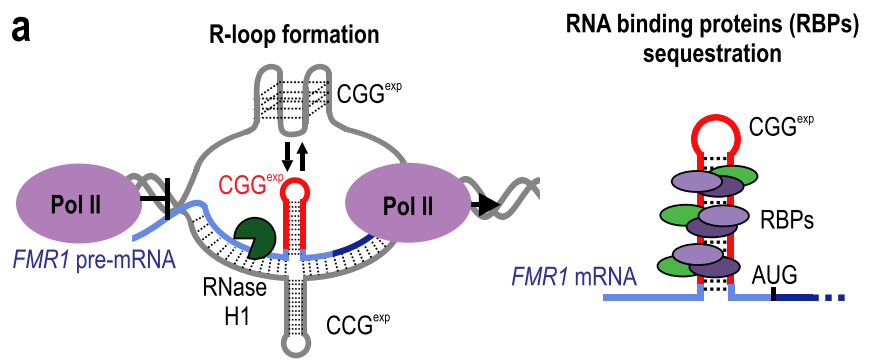

\section{FMRpolyG biosynthesis}

b

RNA-ASO complex formation
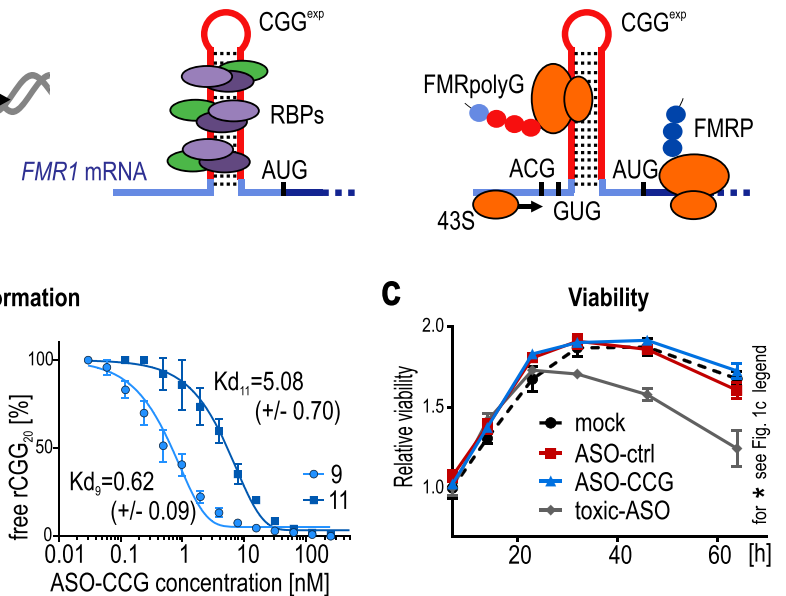

d

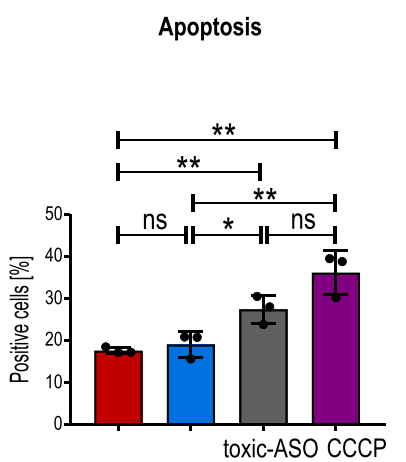

e

Level of miRNAs

f

Splicing of SMN2 minigene

20xCGG 60xCGG 100xCGG

ASO-ctrl $\square$ ASO-CCG
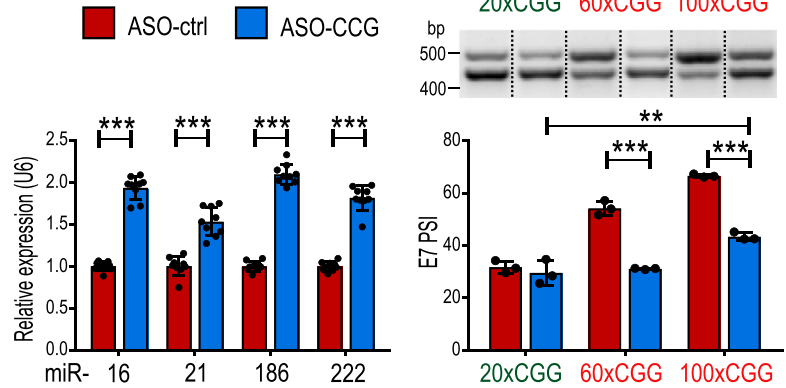

Fig. 1 Binding of ASO-CCG to CGGexp in vitro and in cells reduces the effects of toxic RNA. a Potential molecular pathomechanisms of FXTAS. $\mathbf{b}$ EMSA of the interaction of $\mathrm{rCGG}_{20}$ with ASO-CCGs having 9 and $11 \mathrm{LNA}$ residues. The graph presents means with SDs. $N=3$ independent samples for each concentration. Kd, a dissociation constant [nM]. Light blue/dots, 9 nt; dark blue/squares, $11 \mathrm{nt} \mathrm{ASO.} \mathrm{c} \mathrm{Influence} \mathrm{of} \mathrm{ASOs} \mathrm{(} 9$ nt, $200 \mathrm{nM}$ ) on COS7 cells viability. Graph presents mean of $N=4$ biologically independent samples with the SDs. Black/dots, mock (transfection agent); red/squares, ASO-ctrl; blue/tringles, ASO-CCG; dark gray/diamonds, toxic ASO. For 64-h time point: mock vs ASO-ctrl, $P=0.069$; mock vs ASO-CCG, $P=0.187$; mock vs toxic ASO, $P=0.0004$; ASO-ctrl vs ASO-CCG, $P=0.012$; ASO-ctrl vs toxic ASO, $P=0.001$; ASO-CCG vs toxic ASO, $P=0.0002$. $\mathbf{d}$ Influence of ASOs $(9 \mathrm{nt}$, $200 \mathrm{nM}$ ) on apoptosis of COS7 cells. Graph presents mean of $N=3$ biologically independent samples with the SDs. Dark gray bar, toxic ASO; purple bar, carbonyl cyanide 3-chlorophenylhydrazone (CCCP, positive control). e RT-qPCR quantification of the level of endogenous miRNAs in COS7 cells overexpressing rCGGexp and treated with ASOs $(9 \mathrm{nt}, 200 \mathrm{nM}$ ). Graph presents mean of $N=3$ biologically independent samples (each with $n=3$ technical replicates) with the SDs. $\mathbf{f}$ RT-PCR analysis of SAM68-dependent alternative splicing of exon 7 in SMN2 minigene in COS7 cells overexpressing normal (green) or expanded CGG repeats (red) and treated with ASOs (11 nt, $200 \mathrm{nM}$ ). Graph presents mean of $N=3$ biologically independent samples with the SDs. $100 \times$ CGG ASO-ctrl vs $100 \times$ CGG ASO-CCG, $P=0.00002$. The upper bands, the exon 7 inclusion isoforms; the lower bands, the exon 7 exclusion isoforms. PSI, the percent of spliced in. $\mathbf{b}, \mathbf{f}$ Samples were derived from the same experiment and processed in parallel on different gels. Presented gels were cropped. d-f Red bars, ASO-ctrl; blue bars, ASO-CCG. c-f Two-sided, unpaired Student's $t$ test; ${ }^{\star} P<0.05 ;{ }^{\star \star} P<0.01$; ${ }^{\star \star \star} P<0.001$; ns, non-significant. b-f Source data are provided as a Source Data file.

repeats with normal ( $20 \times \mathrm{CGG})$ and pathogenic $(\sim 60 \times \mathrm{CGG}$ and $\sim 100 \times$ CGG) lengths were overexpressed ${ }^{13,15}$. Overexpression of RNA containing CGG ${ }^{\text {exp }}$ but not normal-length CGG repeats caused significant downregulation of several endogenous mature miRNAs, indicating that DGCR8 was likely sequestered in the latter cells (Supplementary Fig. S1c, d). Furthermore, treatment with ASO-CCG but not with ASO-ctrl significantly increased the levels of mature miRNAs, suggesting that DGCR8 activity was restored in treated cells (Fig. 1e). Similar experiments were performed to assess SAM68 splicing factor activity, by measuring alternative splicing of the exon 7 of SMN2. Cells expressing expanded repeats of $\sim 60$ or $\sim 100$ CGGs but not $\sim 20$ CGGs showed increased exon 7 inclusion in the SMN2 minigene mRNA (Fig. If and Supplementary Fig. S1e, f). Treatment with ASO-CCG but not ASO-ctrl abolished this effect, leading to partial $(100 \times \mathrm{CGG})$ or even full $(60 \times \mathrm{CGG})$ splicing correction
(Fig. If and Supplementary Fig. S1f). By contrast, ASO-CCG treatment did not affect the alternative splicing of SAM68independent event, which proved the specificity of the observed effect (Supplementary Fig. S1g). Overall, these results indicate that the interaction of ASO-CCG with rCGG $^{\exp }$ in cell nuclei can release DGCR8 and SAM68 from pathogenic sequestration, leading to a significant reduction in the effects of toxic RNA in the FXTAS cell model.

ASO-CCG reduces the efficiency of FMRpolyG biosynthesis. Next, we investigated whether ASO-CCG can reduce the level of toxic FMRpolyG. We overexpressed a 100×CGG construct carrying the $5^{\prime}$ UTR sequence of the FMR1 gene containing $~ 100$ CGG repeats (Fig. 2a; artificial FXTAS model) ${ }^{19,23}$. RAN translation of mRNA from this construct leads to the biosynthesis of FMRpolyG fused with GFP (FMRpolyG-GFP). Since FMRpolyG- 
a

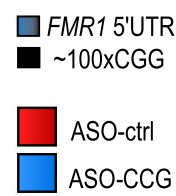

b

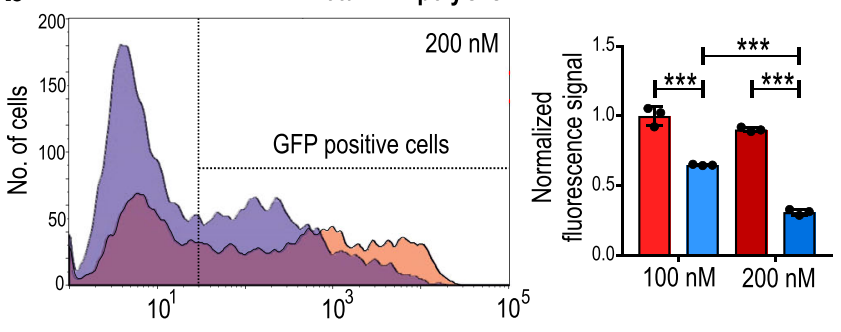

GFP fluorescence signal [log]

C

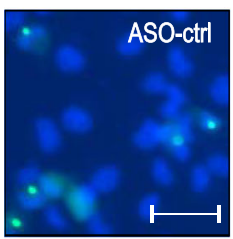

FMRpolyG-GFP inclusions
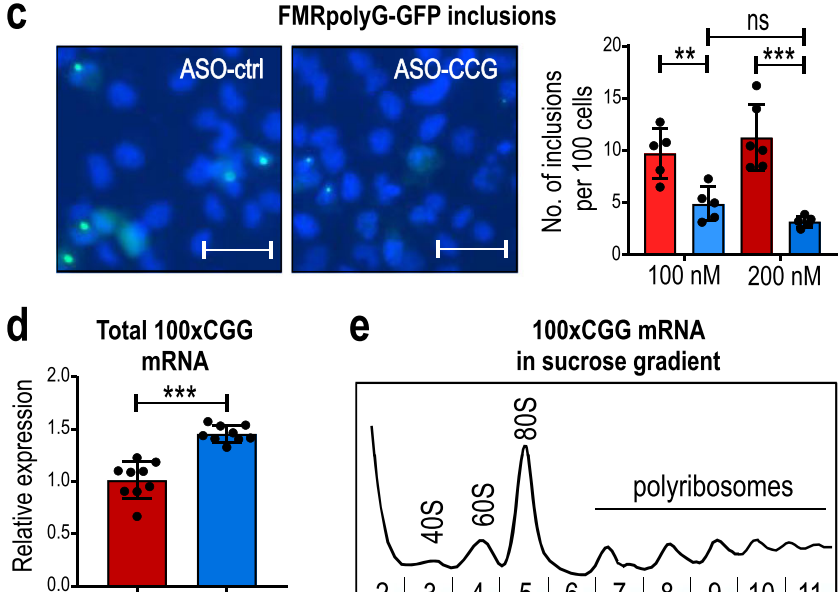

e

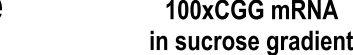

in sucrose gradient
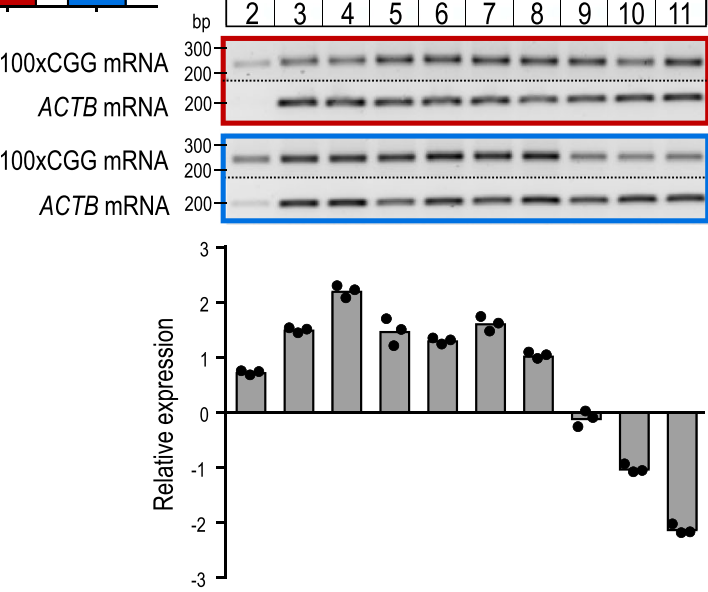

GFP is present in cells in both a soluble and an aggregated form, we used three different methods, based on fluorescence signal quantification, to measure total, aggregated, and soluble forms of this protein ${ }^{23}$. Using a cytometric assay, we showed that the total amount of FMRpolyG-GFP produced in cells expressing $100 \times$ CGG was significantly reduced in cells treated with ASO-CCG compared to that found in the same cells treated with ASO-ctrl, and that this effect was concentration-dependent (Fig. 2b and Supplementary Fig. S2a, b). Using fluorescent microscopy, we found that ASO-CCG treatment resulted in a significant reduction in the number of inclusions of aggregated protein compared to cells treated with ASO-ctrl $(\sim 3$ and $\sim 11$ per 100 nuclei, respectively; Fig. 2c). We also quantified the newly synthesized soluble form of FMRpolyG-GFP as well as FMRP equivalent using fluorescence signal detection after SDS-PAGE
Fig. 2 The effect of ASO-CCG on FMRpolyG biosynthesis and aggregation. a Schematic of the $100 \times$ CGG genetic construct. This construct contains the 5'UTR of the FMR1 gene (blue bars) with 100 CGG repeats (black bar) fused with the GFP coding sequence (green bar). Biosynthesis of the FMRpolyG-GFP via RAN translation starts from the near-cognate start codon, ACG. $\mathbf{b}$ Cytometric quantification of the total level of FMRpolyG-GFP in COS7 cells expressing 100xCGG construct and treated with ASOs (11 nt). The fluorescence signal of GFP-positive cells was measured, excluding dead cells stained with propidium iodide. The histogram presents different FMRpolyG-GFP signal distribution in cells treated with ASO-ctrl (red) or ASO-CCG (blue). $N=3$ biologically independent samples. ASO-ctrl $200 \mathrm{nM}$ vs ASO-CCG $200 \mathrm{nM}, P=$ 0.000003. c Microscopic quantification of FMRpolyG-GFP inclusions in COS7 cells expressing $100 \times$ CGG construct and treated with ASOs (11 nt). Representative images were pseudo-colored and merged; green, GFPpositive inclusions; blue, nuclei stained with Hoechst 33342; scale bars, $50 \mu \mathrm{m} . N=6$ biologically independent samples for ASO-ctrl $200 \mathrm{nM}$ and $N=5$ for other conditions. ASO-ctrl $200 \mathrm{nM}$ vs ASO-CCG $200 \mathrm{nM}, P=$ 0.0003. d RT-qPCR quantification of total mRNA from $100 \times C G G$ construct in COS7 cells treated with ASOs ( $9 \mathrm{nt}, 200 \mathrm{nM}$ ). $N=3$ biologically independent samples (each with $n=3$ technical replicates). e Association of $100 x C G G$ mRNA with polyribosomes. Extracts from COS7 cells transfected with the $100 \times$ CGG construct and $200 \mathrm{nM}$ ASOs (9 nt) were fractionated on a linear sucrose gradient (15-45\%). The total RNA was isolated from the collected fractions representing free mRNAs (the first fraction), monoribosomes, and polyribosomes. Cropped gel presents RTPCR results. The graph presents RT-GPCR results with a mean of $n=3$ technical replicates with the SDs. The reference sample is the corresponding fraction in ASO-ctrl-treated cells and the reference gene is GAPDH. The experiment was repeated 2 times with similar results. b-d Red bars, ASO-ctrl; blue bars, ASO-CCG; light colors, $100 \mathrm{nM}$; dark colors, 200 nM. Graphs present means of indicated $N$ with the SDs. Two-sided, unpaired Student's $t$ test; ${ }^{\star} P<0.05$; ${ }^{\star \star} P<0.01$; ${ }^{\star \star \star} P<0.001$; ns, nonsignificant. b-e Source data are provided as a Source Data file.

separation of cell lysates. ASO-CCG significantly reduced the level of both the soluble FMRpolyG-GFP and FMRP equivalent (Supplementary Fig. S2c).

We then investigated whether ASO-CCG reduced FMRpolyGGFP levels by inducing mRNA degradation or by reducing the mRNA translation rate. Measurement of the total level of rCGG ${ }^{\text {exp }}$ showed significantly higher levels in ASO-CCG-treated cells compared to cells treated with ASO-ctrl ruling out an increase in mRNA degradation (Fig. 2d). Next, to evaluate the dynamics of mRNA translation, we performed sucrose-gradient fractionation of cell lysates after treatment with ASO-CCG. Compared to ASO-ctrl, ASO-CCG treatment increased the abundance of mRNA containing $\mathrm{CGG}^{\exp }$ in the fractions corresponding to free mRNAs, monoribosomes, and light polyribosomes and decreased the association of this mRNA with actively translating heavy polyribosomes (Fig. 2e). This finding suggests that despite the increase in the steady-state level of mRNA containing CGG ${ }^{\exp }$, ASO-CCG interfered with its translation process by binding to $\mathrm{rCGG}^{\exp }$ and preventing its association with polyribosomes, leading to a significant reduction in the FMRpolyG-GFP level.

Short CGG repeat tracts with at least six uninterrupted repeats are present in $\sim 1.5 \%$ of human mRNAs. These repeat tracts may contain as many as 16 pure CGG repeats, and most $(\sim 60 \%)$ are located in $5^{\prime} \mathrm{UTRs}^{28}$. Therefore, we investigated whether ASO-CCG can influence the level and polyribosome association of transcripts containing short CGG repeats. We evaluated endogenous FMR1 and NUB1 mRNAs, which are predicted to contain 12 CGGs repeats. The total level of both transcripts was 
elevated in ASO-CCG-treated cells (Supplementary Fig. S2d) and their distribution in the monoribosome/polyribosome fractions was changed compared to ASO-ctrl-treated cells, with a decreased abundance on heavy polyribosomes (Supplementary Fig. S2e). These results suggest that ASO-CCG influences the total level of transcripts containing not only expanded but also short CGG repeats and, possibly through binding to the $5^{\prime}$-end of these mRNAs, may decrease their translation rate.

ASO-CCG increases the transcription of the FMR1 locus and reduces the efficiency of FMRP biosynthesis. ASO-CCG increased the level of exogenous mRNAs containing CGGexp and transcribed from the genetic construct in the artificial FXTAS model. Therefore, we asked whether ASO-CCG exerts a similar effect on endogenous FMR1 mRNA and pre-mRNA in human fibroblasts. Moreover, it had been previously shown that R-loops in 5'-parts of other genes may reduce transcription efficiency ${ }^{29}$; therefore, we also wanted to test whether ASO-CCG affects R-loop formation and influences FMR1 transcription. We used four fibroblast cell lines derived from two men with a normal range of CGG repeats (one with 20 and the other with 31 CGGs) and from two women carrying alleles with CGG ${ }^{\exp }$ (one with one normal and one CGG ${ }^{\exp }$ allele and the other with two alleles containing CGG ${ }^{\exp }$ ). ASO-CCG significantly increased the total level of not only FMR1 mRNA but also FMR1 pre-mRNA in three cell lines (Fig. 3a and Supplementary Fig. S3a). Only in cells carrying short, 20 CGG repeat tract $\left(\mathrm{CGG}^{\text {norm}} /\right.$ - (1)) treatment with ASO-CCG did not affect the level of mRNA and pre-mRNA. The observed effect could be partially caused by ASO-CCG-based prevention of R-loop formation within the promoter region of $F M R 1$ and the subsequent increase in transcription efficiency. R-loops are structures sensitive to RNase H1, which cleaves RNA molecules within RNA/ DNA duplexes and destroys these structures. Therefore, we measured the level of FMR1 mRNA and pre-mRNA in the same fibroblasts upon the reduced level of RNase H1. In three tested cell lines treated with siRNA against RNase $\mathrm{H} 1$, the increase in the FMR1 mRNA level induced by ASO-CCG was greatly diminished compared to control siRNA-treated cells, while the increase in the pre-mRNA level was no longer statistically significant (Fig. 3a and Supplementary Fig. S3a). These data suggest that ASO-CCG may enhance the transcription efficiency of the FMR1 locus with longer CGGs by targeting R-loops and changing their thermodynamic stability.

The formation of RNase H-, RNase A-, and DNase-sensitive R-loops in the FMR1 5'UTR with 100 CGGs $\left(\mathrm{rCGG}_{100}\right)$ was further confirmed in in vitro transcription system using both linear and circular DNA templates (Supplementary Fig. S3b). Our in vitro studies showed that the synthesis of $\mathrm{rCGG}_{100}$ significantly increased in the presence of RNase $\mathrm{H}$ and in the presence of ASO-CCG compared to ASO-ctrl-treated samples (Fig. 3b), as was observed in cellula (Fig. 3a), which suggests that ASO-CCG influences on the transcription efficiency via modulation the stability of R-loop structure. The direct interaction between Rloops and ASO-CCG was further confirmed using fluorescently labeled ASO-CCG-Cy3 in in vitro transcription in the absence of RNase H (Fig. 3c). On the other hand, the presence of RNase H significantly reduced the amount of R-loop structures. As we expected, there was also a fraction of ASO-CCG-Cy3 which bound to a sense strand of DNA template containing CGGexp (Fig. 3c and Supplementary Fig. S3c). This binding may abolish the stabilizing effect of interaction between RNA and DNA structures formed by CGG repeats ${ }^{8}$ (see Fig. 1a). Therefore, these experiments showed the ability of ASO-CCG to invade the R-loop structure, which is sensitive to RNase H, and confirmed the positive effect of the oligonucleotide on the transcription efficiency of $\mathrm{rCGG}_{100}$.

To further assess the degree to which the observed increase in total FMR1 mRNA in ASO-CCG-treated FXTAS cells is related to nuclear processes (e.g., transcription or nuclear retention of mRNA) or cytoplasmic processes (e.g., mRNA stability) we performed nucleocytoplasmic fractionation. Upon ASO-CCG treatment, the nuclear level of FMR1 mRNA was elevated only in one cell line with a normal CGG repeat number and in all CGG $^{\text {exp }}$-carrying fibroblasts, whereas its cytoplasmic level was considerably increased in all tested cell lines (Fig. 3d and Supplementary Fig. S3e). Our findings suggest that ASO-CCG affects transcription of $F M R 1$ alleles, especially the one carrying CGG $^{\exp }$ (Fig. 3a for pre-mRNA and d for nuclear fraction). It may also impact the nuclear retention, nucleocytoplasmic transport, subcellular localization, and stability of FMR1 mRNA. These processes jointly may lead to a pronounced increase in FMR1 mRNA level in the cytoplasm regardless of the CGG repeat length (20-90 repeats). Since an increase in the level of FMR1 pre-mRNA and nuclear mRNA is relatively low in ASO-CCG-treated cells we believe that the significant increase of FMR1 mRNA in the cytoplasm is caused mainly by the elevated stability of mRNA, perhaps due to reduced efficiency of translation of both FMRpolyG and FMRP. A similar effect was previously described for many other genes ${ }^{30,31}$.

The above-mentioned results from the artificial FXTAS cell model showed that despite the increase in $\mathrm{rCGG}^{\exp }$ level, translation of the mRNA into FMRP equivalent was reduced in the presence of ASO-CCG (Supplementary Fig. S2c). Since FMR1 mRNA level was also increased in human fibroblasts, we next investigated whether the biosynthesis of FMRP was affected in these cells. The western blot results showed unchanged steadystate level of FMRP upon ASO-CCG treatment for five out of six cell lines (four with a normal number of CGG repeats and one with 100 CGGs), despite of 1.5-2 times increase of its mRNA level (Fig. 3a, d, e and Supplementary Fig. S3d). Only one cell line carrying $\mathrm{CGG}^{\exp }$ showed a significantly lower level of FMRP (Fig. 3e). Our results suggest that ASO-CCG indeed reduces the efficiency of FMRP biosynthesis; however, perhaps due to the increased level of its mRNA, the steady-state level of protein is not affected.

ASO-CCG reduces the formation of FMRpolyG inclusions and improves motor performance in an FXTAS mouse model. The promising results obtained in cellular models of FXTAS encouraged us to evaluate the therapeutic potential of ASO-CCG in an animal model and to test whether a reduction of CGGexp. induced toxicity would be associated with an improvement of motor functions in vivo. We selected a bitransgenic, inducible P90CGG mouse model in which the expression of a transgene containing CGG ${ }^{\exp }$ is under the control of a prion gene promoter with an expression pattern predominantly in the central nervous system and is activated by doxycycline (DOX) induction ${ }^{32,33}$. These mutants show a robust and controllable expression of CGG $^{\exp }$ in neural cells and faithfully model FXTAS phenotypes including intranuclear inclusions and motor disturbance without signs of overt transgene toxicity or increased animal mortality, thus providing an excellent system to test the effectiveness of ASO-CCG in vivo ${ }^{32,33}$.

The main problem with the potency of ASOs in vivo is their bioavailability. Therefore, we first investigated whether ASO-CCG could be efficiently delivered into cells in the absence of a carrier. Fluorescently labeled oligomers added directly to the cell medium were detected within the majority of cells, and an increase in the concentration of approximately twofold was needed to obtain 
a
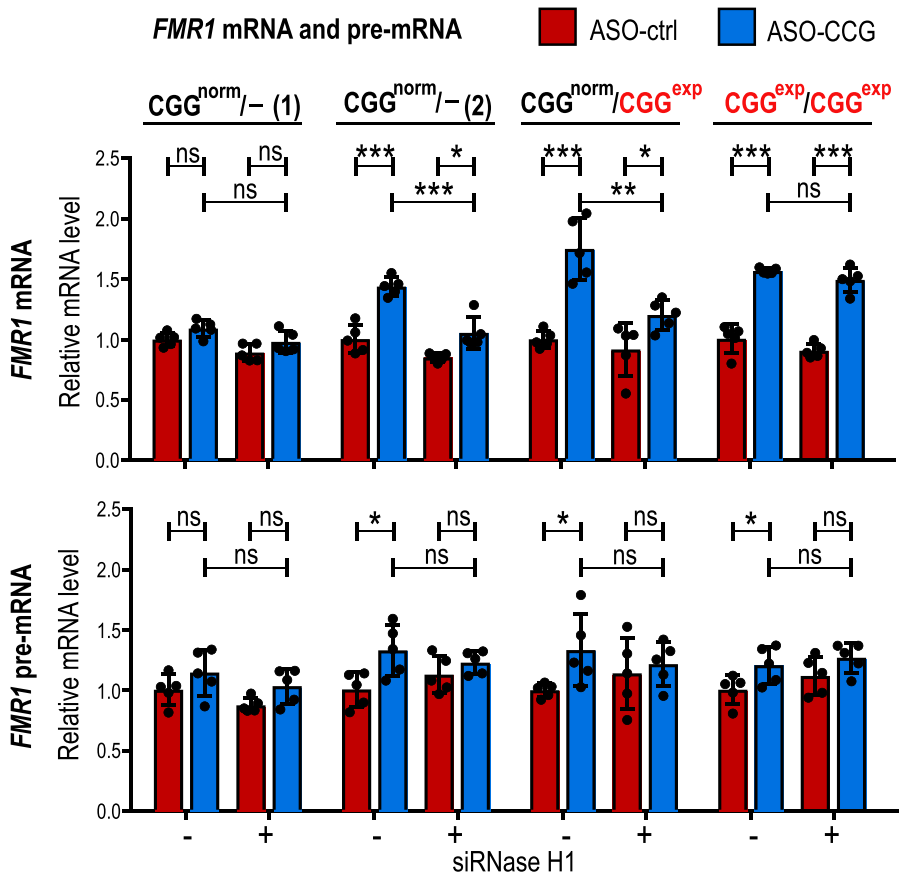

b

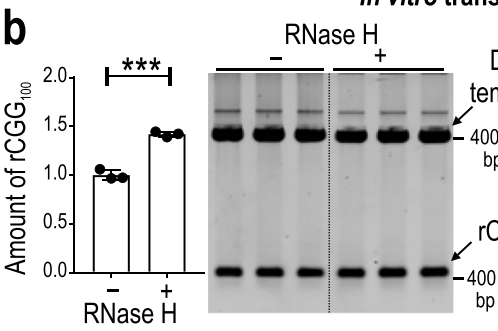

In vitro transcription efficiency

C Effect of ASO-CCG-Cy3 binding
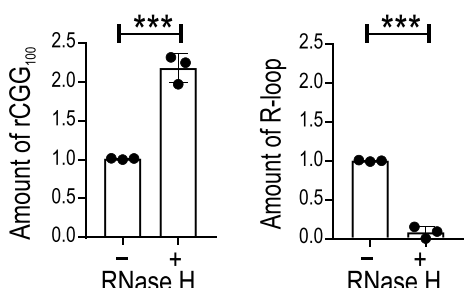

RNase ${ }^{+} \mathrm{H}$
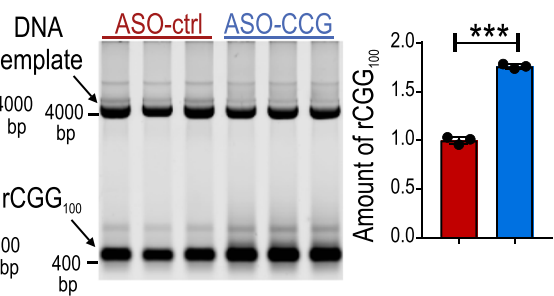

d
FMR1 mRNA

cellular distribution
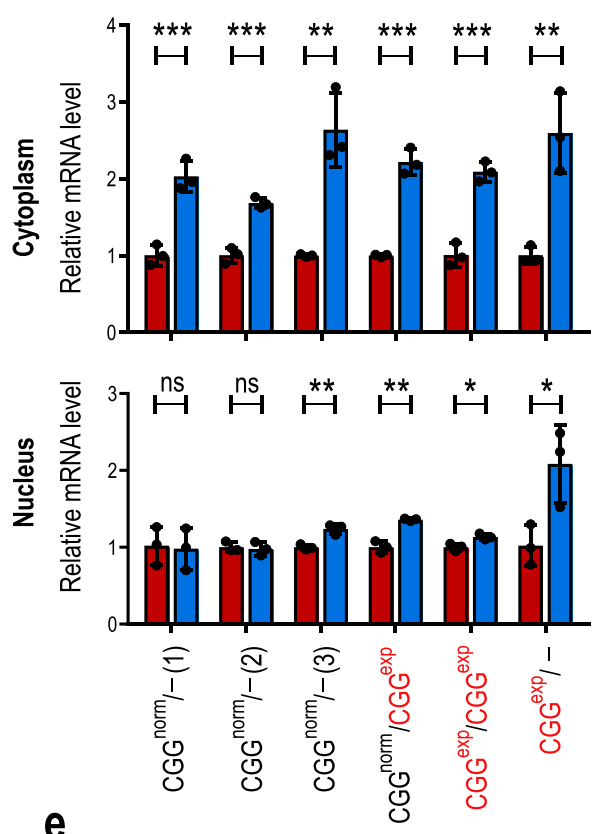

e

Level of FMRP

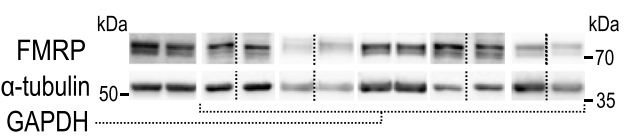

GAPDH

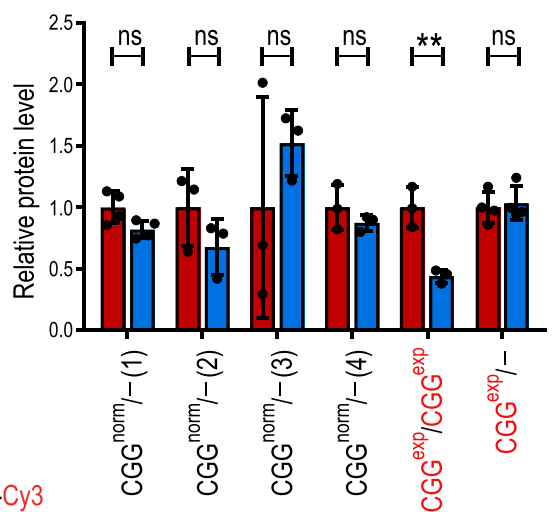

Fig. 3 The effect of ASO-CCG on the FMR1 pre-mRNA, mRNA, and FMRP levels in FXTAS patient-derived fibroblasts. a Analysis of the total FMR1 mRNA and pre-mRNA levels in fibroblasts treated with ASOs. Fibroblasts were transfected with siRNA against RNase H1 or control siRNA and with ASOs ( 9 or $11 \mathrm{nt}, 200 \mathrm{nM}$ ). Graphs present RT-qPCR results for $N=5$ biologically independent samples. $\mathbf{b}$ Increase in the efficiency of in vitro transcription in the presence of ASO-CCG or RNase $\mathrm{H}$. $\mathrm{rCGG}_{100}$ signal was measured for $\mathrm{N}=3$ independent samples. c Correlation between R-loop formation and efficiency of transcription of RNA containing $\mathrm{CGG}_{100}$. Graphs present quantification of the fluorescent signal coming from fluorescently labeled ASO-CCG-Cy3 bound to either $\mathrm{rCGG}_{100}$ or $\mathrm{R}$-loop structure for $\mathrm{N}=3$ independent samples. The area of the gel marked with a red box was exposed to higher laser power strength and the result is presented below the main gel. DNA template was the same as in $\mathbf{b}$, but the observed signal came only from partially single-stranded DNA in the region of CGG/CCG repeats or R-loop bound with ASO-CCG-Cy3. d Cellular distribution of FMR1 mRNA in fibroblasts treated with ASOs (9 nt, $200 \mathrm{nM}$ ). Graphs present RT-qPCR results for the cytoplasmic and nuclear fractions for $N=3$ biologically independent samples. Results were normalized to GAPDH. e Changes in the steady-state level of FMRP after treatment with ASO-CCG. Fibroblasts were transfected with $200 \mathrm{nM}$ ASOs ( 9 or $11 \mathrm{nt}$ ). The graph presents western blot results for $N=4$ biologically independent samples (CGGnorm/- (1) and CGGexp/-) and N=3 (other cell lines). a-e Graphs present means of indicated N with the SDs. a, b, d, e Red bars, ASO-ctrl; blue bars, ASO-CCG. a, d, e The used cell lines contained one allele with a normal CGG repeat length (CGGnorm/-; lines (1), 20 CGG repeats; (2), 31 CGG repeats; (3) and (4)); one allele with a normal CGG repeat length and one allele with CGGexp (CGGnorm/CGGexp); two alleles with CGGexp (CGGexp/ CGGexp) and one allele with CGGexp (CGGexp/-). b, c, d Gels were cropped. a-e Two-sided, unpaired Student's $t$ test; ${ }^{\star} P<0.05 ;{ }^{\star \star} P<0.01 ;{ }^{\star \star \star} P<$ 0.001 ; ns, non-significant. Source data are provided as a Source Data file. 
a ASO distribution in cells

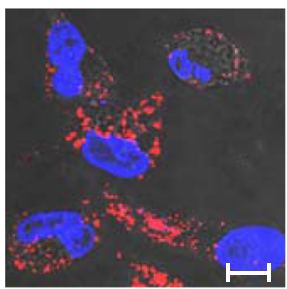

C

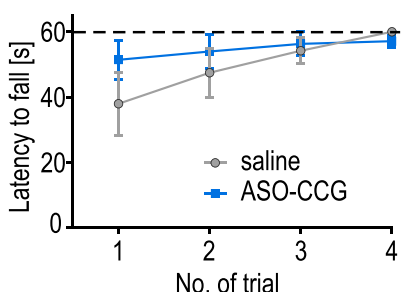

b Experimental design

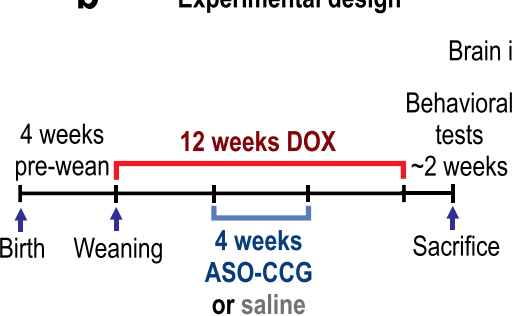

Catheter tube

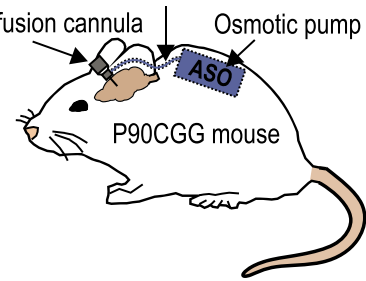

d Constant speed rotarod

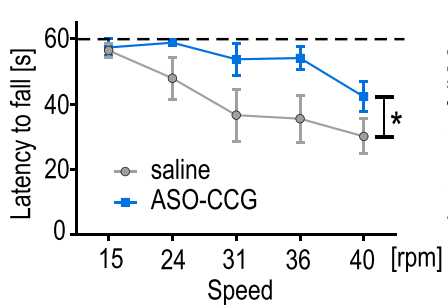

FMRpolyG inclusions
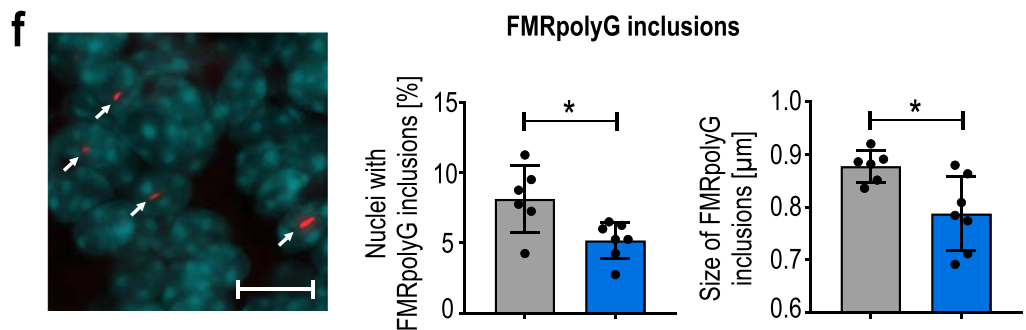

e Accelerating rotarod

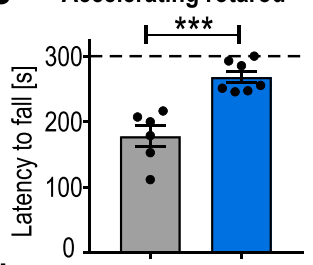
saline ASO-CCG

Fig. 4 The effect of ASO-CCG on behavioral and molecular phenotypes in the FXTAS mouse model. a Representative image showing the distribution of ASO-CCG-Cy3 (red; 200 nM) in COS7 cells. ASO entered cells in the absence of a carrier. Blue, stained nuclei; scale bar, $20 \mu \mathrm{m}$. The experiment was repeated two times with similar results. b Experimental design for DOX induction (red) and ASO-CCG delivery (blue) into P90CGG mice. The contents of the osmotic pump (ASO-CCG (11 nt) solution or saline) were delivered into the right lateral ventricle via intracerebroventricular infusion $(\sim 550 \mu \mathrm{g}$ of ASO-CCG per mouse). c Training session for the rotarod test. Mice treated with saline and ASO-CCG were subjected to a 3-day rotarod test starting with a training session performed at $15 \mathrm{rpm}$ constant speed (day 1). No differences were observed between groups. $\mathbf{d}$ Constant speed rotarod test. On day 2 , the above-mentioned mice were subjected to a constant speed rotarod test at different speeds ( $x$ axis; rpm, revolution per minute). The ASO-CCG-treated group stayed longer on the rod, $P=0.0323$. e Accelerating rotarod test. On day 3, mice were subjected to an accelerating rotarod test (4-40 rpm ramp). The ASO-CCG-treated group showed better performance on this test (longer latency to fall), $P=0.0003$. Dashed line, maximum cutoff $\mathbf{c}$, $\mathbf{d} 60 \mathrm{~s}$, e $300 \mathrm{~s}$. f Quantification of FMRpolyG foci in P90CGG mice. A representative image of FMRpolyG staining, sections containing cerebellar lobule X of P90CGG mice was stained using the 8FM antibody specific for FMRpolyG (scale bar, $10 \mu \mathrm{m}$ ). For the ASO-CCG-treated group, the percentage of the nuclei with positive staining for FMRpolyG (red) was lower $(P=0.0161)$ and the size of the inclusions they contain (indicated by a white arrow) were smaller $(P=0.0146)$ than that of the saline-treated group. Graphs present means with SDs. a, $\mathbf{f}$ Images were pseudo-colored and merged. c, $\mathbf{d}$ Two-way repeated-measures ANOVA (treatment effect): ${ }^{\star} P<0.05$. e, f Two-sided, unpaired Student's $t$ test: ${ }^{\star} P<0.05$; ${ }^{\star \star \star} P<0.001$. c-e Graphs present means with SEMs. c-f Gray dots/bars, saline-treated animals $(N=6)$; blue squares/bars, ASO-CCG treated animals $(N=7)$. Source data are provided as a Source Data file.

an inhibitory effect on RAN translation comparable to that of transfection with lipophilic carriers (Fig. 4a and Supplementary Fig. S4a). Therefore, we assumed that unassisted uptake of ASO-CCG into neurons in vivo was feasible when delivered via intracerebroventricular infusion.

To check the effect of ASO-CCG on FXTAS-specific symptoms in P90CGG mice, we designed a specific treatment procedure featuring an intracerebroventricular infusion of either ASO-CCG or saline as a control. Our previous study showed that 12 weeks of DOX-induced expression of CGG ${ }^{\exp }$ is sufficient to obtain a characteristic FXTAS-specific phenotype, e.g., the formation of FMRpolyG inclusions in neural cells and motor deficits, that is stable after cessation of transgene induction. Therefore, we adopted a $4+4+4$ treatment schedule (Fig. 4b), with ASO-CCG infusion starting after 4 weeks of DOX induction. In the ensuing 4 weeks, mice received both DOX and ASO-CCG followed by 4 additional weeks of the only DOX. Then, animals were subjected to motor behavior tests, and, finally, about 17 days after the last DOX exposure brain tissues were collected and molecular assays were performed.
We used rotarod tests to assess the motor performance of treated animals. In our previous study, while P90CGG DOXcontrol mice performed close to maximum cutoff levels on the rotating rod, significant deficits were present in the P90CGG mice upon 12 weeks of DOX induction both on the constant high speed and the accelerating rotarod. Moreover, a shorter 8-week transgene expression was not sufficient to drive these deficitis ${ }^{33}$. We therefore now, in the ASO-treated FXTAS model under 12 weeks of DOX induction and comparable test conditions performed a series of rotarod tests over three days comprising of a training session, a constant speed test session, and an acceleration ramp test session. In the training session, at low speed, ASOtreated mice showed no differences in the latency to fall off the rod compared to saline-treated mice (Fig. 4c), suggesting a comparable acclimation level in both groups at the start of the testing session on day 2. However, in the constant speed rotarod test, the performance of ASO-treated mice was significantly better than that of their saline-treated counterparts especially at higher speeds (Fig. 4d). The performance of ASO-treated mice was also better on day 3 , as they remained on the accelerating rod 
significantly longer than saline-treated mice (Fig. 4e). The rotarod test results were not affected by differences in the body weights of the tested mice (Supplementary Fig. S4b).

Then, we investigated the molecular mechanism underlying the behavioral improvements following ASO-CCG treatment. Intranuclear inclusions, the hallmark of FXTAS pathology, are considered to reflect a neurotoxic action, and we previously reported that large numbers of these inclusions were present in cerebellar lobule $\mathrm{X}^{32,33}$. We quantified the number of intranuclear inclusions in the granule cell layer of this brain structure using an antibody specific for the FMRpolyG peptide (Fig. 4f, left and Supplementary Fig. S4c). Not only the percentage of nuclei with positive staining for FMRpolyG but also the average size of the inclusions was significantly decreased in ASO-treated brains (Fig. 4f, right).

\section{ASO-CCG ameliorates the transcriptome changes of FXTAS} mouse brain. To gain deeper insight into the molecular changes induced by ASO-CCG treatment, we conducted RNA-seq analyses of the striatum, one of the brain structures with the highest level of transgene expression ${ }^{32}$. Approximately 50-70 million reads were obtained for each sample, and differential analysis of $\sim 17,000$ genes was performed for three groups of animals: bitransgenic P90CGG mice expressing CGG $^{\exp }$ and treated with either (1) ASO-CCG or (2) saline and (3) monotransgenic mice harboring the transgene containing CGG ${ }^{\exp }$ but not expressing it (the control group; all mice were treated with DOX). The expression of 417 genes was significantly changed (adjusted $P$ value $<0.05)$ in P90CGG mice treated with saline compared to control mice (Supplementary Data S1). These CGG ${ }^{\exp }$-dependent changes can be considered FXTAS-related. Therefore, we expected that treatment with ASO-CCG could restore the expression of at least some of these genes toward the level observed in the control group. Indeed, we found a significant global correction of the expression of FXTAS-related genes in ASO-CCG-treated mice, as evidenced by the negative Pearson correlation $(r=-0.75 ; P<0.001)$ (Fig. 5a, upper panel and Supplementary Fig. S5). The expression level of 57 of the 417 genes was completely restored, and the correction of most of the others was substantial. Then, we performed gene ontology analysis to obtain better insight into the functions of abnormally expressed FXTAS-related genes (Supplementary Data S2). Among the downregulated genes, genes involved in translation (mostly structural constituents of the ribosome), amide biosynthesis, and oxidation-reduction processes were significantly enriched (adjusted $P$ value $<0.001$ ). The enriched pathways were nonsensemediated RNA decay, respiratory electron transport, ribosomal scanning, and start codon recognition (Fig. 5a, gray bars). On the other hand, among the upregulated genes, genes linked with nervous system development and playing a role in transcription coregulator activity were significantly enriched. Importantly, all of these gene ontology categories were mainly unchanged in ASO-CCG-treated mice compared to control mice (Fig. 5a, blue bars). These results again suggest significant rescue of the molecular phenotype observed at the transcriptome level after ASO-CCG treatment.

Next, to examine the off-target effects of ASO-CCG in vivo, we performed RNA-seq analysis of both the striatum as well as the cortex, in which the expression of CGG ${ }^{\exp }$ is very low but the concentration of ASO-CCG is expected to be high due to the direct contact of this structure with the cerebrospinal fluid where the ASO was infused into. Among the $\sim 16,000$ analyzed genes, $\sim 150$ contain at least six uninterrupted CGG repeats in exonic sequences, mainly in the $5^{\prime}$ UTRs. On a global scale, the expression of this group of genes was significantly elevated in
ASO-treated mice compared to saline-treated mice (Fig. 5b and Supplementary Fig. S6a, b; Supplementary Data S3 and S4), confirming our previous observation in cell-based models of increased levels of mRNAs containing CGG repeats. Gene ontology analysis showed slight but statistically significant enrichment of immune system-related genes, suggesting that ASO-CCG induces an immune response in the brain cortex (Supplementary Table S1).

Therefore, observations coming from RNA-seq analysis pushed us to study both the off-target effect and immune system-related toxicity of ASO-CCG on a protein level. As potential off-targets, we chose genes containing CGG repeats in their 5'UTRs and exhibiting an elevated mRNA level in the ASO-CCG-treated brain tissue, according to the RNA-seq data. Protein products of these genes were analyzed using western blot. The level of none of them was changed in a result of ASO treatment (Fig. 5c). Moreover, we checked the level of these proteins in two fibroblast cell lines with normal and expanded CGG repeats in FMR1 gene. We found that the level of only two out of four tested proteins, namely QKI (Protein quaking) and VKORC1L1 (Vitamin K epoxide reductase complex subunit 1-like protein 1), was significantly reduced, but in solely one ASO-CCG-treated cell line (Supplementary Fig. S6c, d). Treatment with ASO-CCG in either type of cell did not exert any effect on the level of DAZAP1 (DAZ-associated protein 1), whose mRNAs contain 14 CCG repeats within the $5^{\prime} \mathrm{UTR}$.

Immune system-related toxicity markers were analyzed on the hippocampus and cortex derived from saline or ASO-CCGtreated mice. Western blot revealed that a marker of gliosis, glial fibrillary acidic protein (GFAP) was present at similar levels in both groups of mice, in spite of an increase at mRNA levels detected from ASO-CCG-treated brains (Fig. 5d, e). Since the transformation of full-length GFAP into shorter form is considered as a sign of glial injury ${ }^{34}$ we also quantified GFAP's proteolytic fragment level and observed an increase, however, not statistically significant. Similarly, the increase at the mRNA levels of CD68 (macrosialin), a marker of activated microglia, was not observed at the protein level. The protein levels of allograft inflammatory factor 1 (AIF1), another marker of microglia, tumor necrosis factor $\alpha$ (TNF- $\alpha$ ), a marker of inflammation, as well as protein phosphatase 1 regulatory subunit $1 \mathrm{~B}$ (PPP1R1B), a marker of medium spiny neurons, were also unchanged in ASO-CCG-treated brains (Fig. 5d, e and Supplementary Fig. S6e, f). Overall, our investigation of potential toxicity markers shows no evidence of prominent adverse effects related to ASO-CCG treatment.

\section{Discussion}

Antisense strategies using steric blockers are being widely tested as therapeutics for a range of human diseases, including neurodegenerative disorders ${ }^{35}$. This class of ASO binds to its targeted RNA sequence and modifies the maturation or metabolism of the targeted RNA without inducing transcript degradation. Recently, two ASO steric blockers that modify the splicing of specific exons were approved for the treatment of Duchenne muscular dystrophy (DMD) and spinal muscular atrophy (SMA) ${ }^{36,37}$. Short ASOs composed exclusively or predominantly of LNA units were successfully used to repress miRNA activity in animal models, and their activity against the hepatitis $\mathrm{C}$ virus is being tested in clinical trials ${ }^{38,39}$. The advantages of using LNAs are the low toxicity, resistance to cellular nucleases, and extremely high thermodynamic stability of the duplex formed with complementary RNA. This allows the use of very short ASO sequences that can penetrate into cells and tissues in the absence of specific carriers via a receptor-based endocytosis mechanism known as 


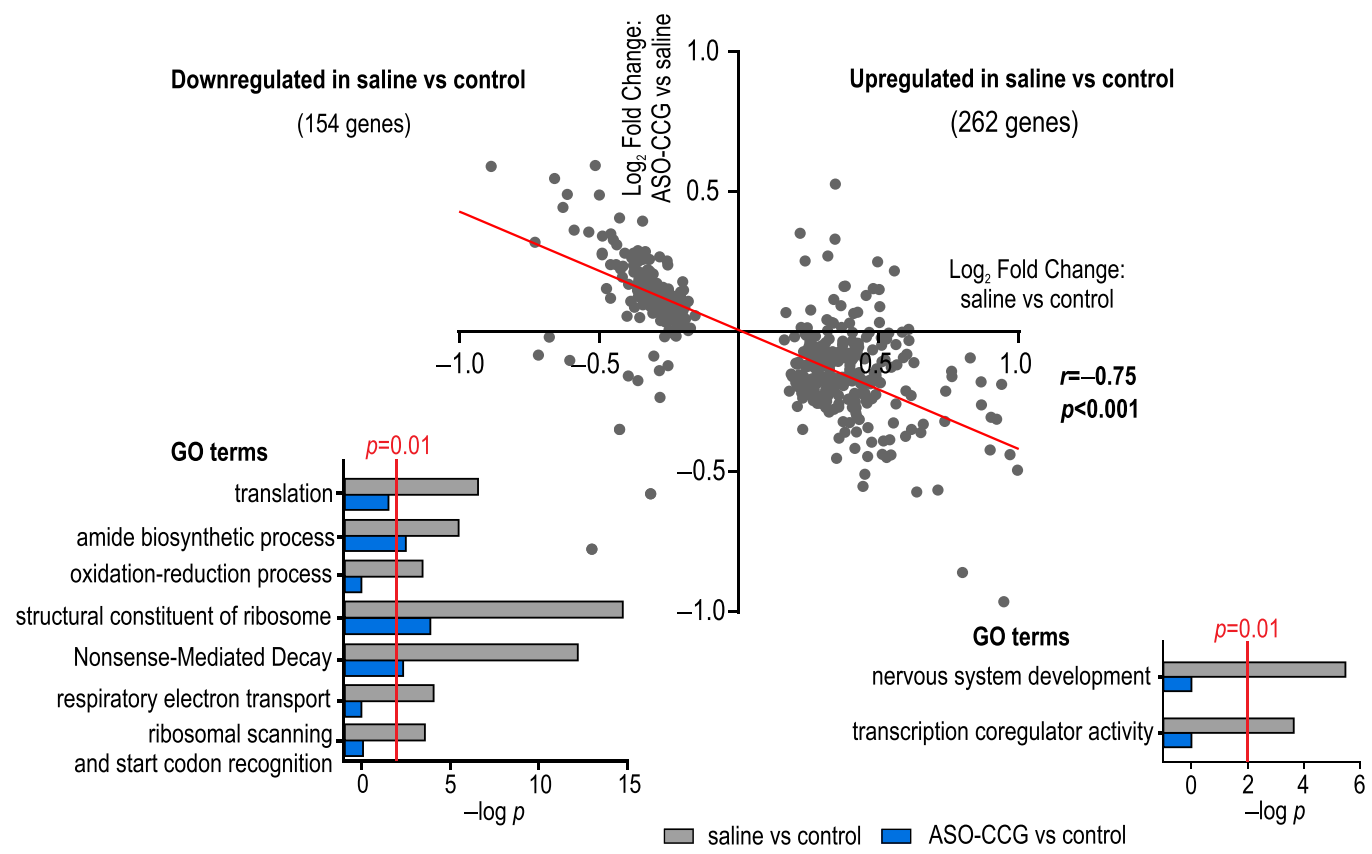

b Expression of genes
with CGG repeats

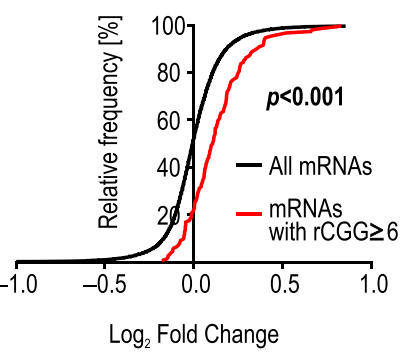

saline ASO-CCG

d

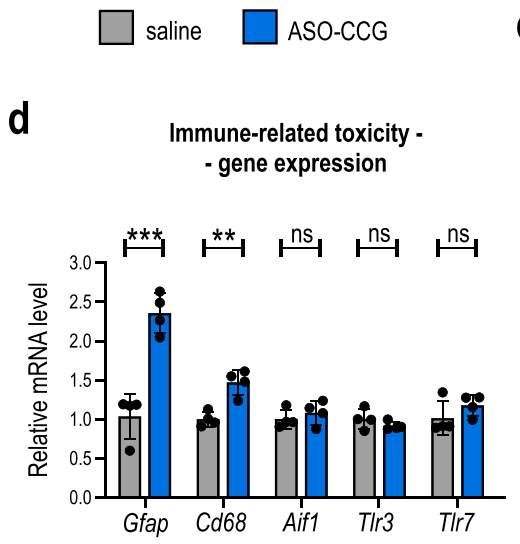

e
C

Fmr1 (6xCGG)
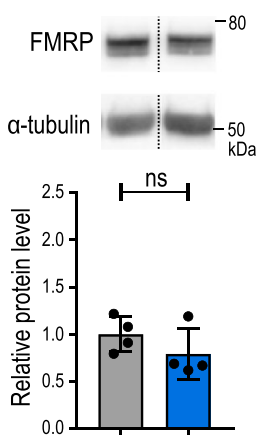

Proteins from CGG containing mRNAs

Qki (7xCGG) Slc40a1 (6xCGG)

Vkorc111 (8xCGG)

QKI
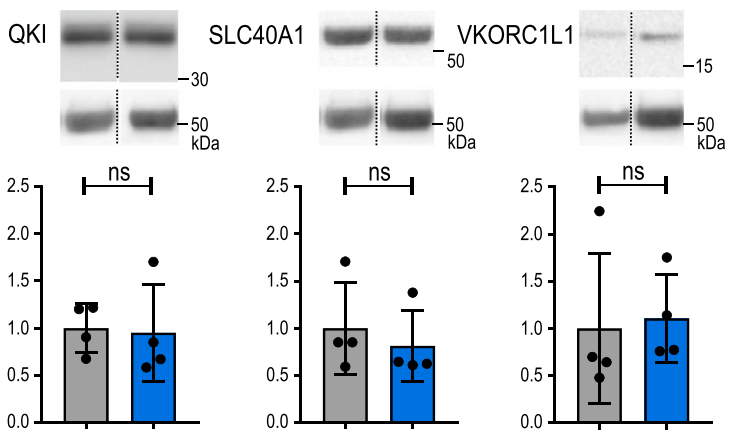

Immune-related toxicity - level of proteins
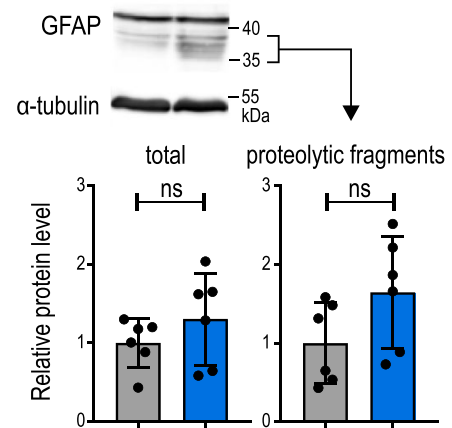

CD68
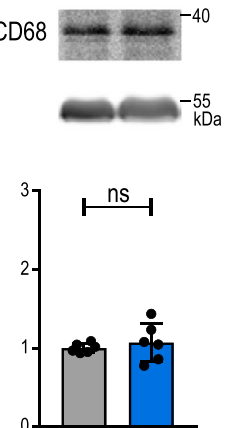

AIF1
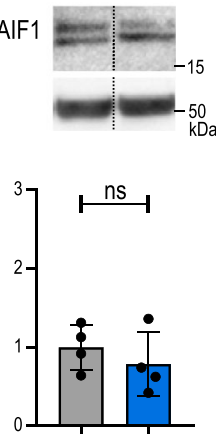

gymnosis $^{39}$. We showed that ASO-CCG also exhibits these features (Fig. 4a). Moreover, our results suggest that ASOs in which the number of LNA units is a multiple of $3(6,9,12$, etc.) bind even more tightly to targeted triplet repeat RNAs because of the stabilizing effect of stacking interactions between the bases of consecutive ASOs (Fig. $1 \mathrm{~b}$ and Supplementary Fig. S2b). We believe that this observation may also help to design antisense therapeutic strategies for other 3-6 nucleotide repeat expansion diseases.

Here, we showed that ASO-CCG affects multiple stages of mutant FMR1 gene expression, e.g., transcription, nuclear metabolism, and translation. The increased levels of target mRNAs containing both short and expanded CGG repeats upon ASO-CCG treatment suggest the involvement of this compound 
Fig. 5 Beneficial and adverse effects of ASO-CCG (11 $\mathbf{n t )}$ at a molecular level in an FXTAS mouse model. $\mathbf{a}$, $\mathbf{b}$ DE, results based on differential gene expression analysis of the RNA-seq data. adj.P.Val, $P$ value generated using moderated $t$-statistic and adjusted for multiple testing using Benjamini-Hochberg's method. a Plot, DE for striatum of control, saline- or ASO-CCG-treated P90CGG mice $(N=4$ per group). On the $x$ axis, the log 2 fold change in the expression levels of the 416 genes (one outlier gene, Gm20425, was excluded from analysis), that were significantly changed (adj.P.Val < 0.05) in saline-treated P90CGG mice compared to control mice is plotted. On the $y$ axis, the $\log _{2}$ fold change in the gene expression levels of the same genes compared between ASO-CCG- and the saline-treated group is plotted. A general restoration of the expression levels towards the levels in control mice was observed in the ASO-CCG-treated group (red line, negative Pearson correlation with $r=-0.75$ and $P<0.001$ ). Bar graphs, gene ontology analysis of genes extracted from DE for striatal tissue according to the following parameters: average expression value $>1$ and adj.P.Val $\leq 0.05$. Significantly upregulated and downregulated genes were analyzed for the saline vs control (gray) and ASO-CCG vs control groups (blue). Red line, $P=0.01$ in Fisher's exact test with Bonferroni correction for multiple testing. b Analysis of expression changes in genes containing CGG repeats in ASO-CCG-treated P90CGG mice. DE for the cortex of saline- or ASO-CCG-treated P90CGG mice ( $N=4$ for each group). A global comparison of gene expression between these two groups of mice showed a significant increase in the expression of 22 genes containing at least 6 CGG repeats (red line) compared to all analyzed genes (black line; $P<0.001$, two-tailed Mann-Whitney test). Moreover, in the list of genes showing significant expression changes between these two groups (adj.P.Val < 0.05), genes containing $\geq 6$ CGG repeats were enriched 13-fold relative to the expected level. c Steady-state level of proteins encoded by genes carrying short CGG repeats in 5'UTRs in the cortex of saline- and ASO-CCG-treated P90CGG mice. $N=4$ animals. The CGG repeat length is specified for each gene. $\mathbf{d}$ RT-qPCR of immune system-related genes in the cortex of P90CGG mice. $N=4$ animals. e Steady-state level of immune systemrelated toxicity markers in P90CGG mice. Hippocampus: GFAP, CD68, N=6 animals. Samples were derived from the same experiment and processed in parallel on different gels. Cortex: AIF1, $N=4$ animals. c, e Western blot. All blots were cropped. c-e Gray bars, saline-; blue bars, ASO-CCG-treated animals. Graphs present means of indicated $N$, with the SDs. Two-tailed unpaired Student's $t$ test, ${ }^{\star \star} P<0.01 ;{ }^{\star \star \star} P<0.001 ; P>0.05$, ns, non-significant. a-d Source data are provided as a Source Data file.

in cotranscriptional processes, such as R-loop formation (Fig. 3a-c and Supplementary Fig. S3a, c). A previous study suggested that $\mathrm{CGG}^{\exp }$ within the FMR1 locus either increase the formation efficiency of R-loops or positively affect their thermodynamic stability ${ }^{6,8}$. Here, we showed that the presence of RNase H-sensitive R-loops within the FMR1 locus decreases $F M R 1$ transcription both in vitro and in vivo (Fig. 3a-c), perhaps due to the formation of a steric barrier for RNA polymerase. Furthermore, we found that ASO-CCG reduces R-loop formation and/or their stability and significantly increases FMR1 transcription. The ASO-CCG-induced increase in FMR1 pre-mRNA and mRNA is significantly milder in FXTAS-derived cells with RNase $\mathrm{H} 1$ deficiency, which generally reduces the negative effect of R-loops on transcription (Fig. 3a, b and Supplementary Fig. S3a). R-loops within CGG ${ }^{\exp }$ loci may cause DNA breakage and induce the DNA damage response in FXTAS cells ${ }^{6}$, possibly leading to neuronal death. Therefore, ASO-CCG may also exert a protective effect against double-stranded DNA breaks by inhibiting R-loop formation and/or reducing their durability. Beside cotranscriptional events, several posttranscriptional processes may also induce an increase in the FMR1 mRNA level due to ASO-CCG treatment. One potential mechanism could be increased efficiency of nucleocytoplasmic transport, what we previously showed for mRNA containing CUG repeat expansion ( $\mathrm{rCUG}^{\mathrm{exp}}$ ) after treating muscles of myotonic dystrophy mice with short LNA-composed ASOs targeting CUG repeats ${ }^{40,41}$. Other mechanisms could be linked to the increased stability of mRNA. According to recent findings, slowing down the ribosome movements or lowering the density of ribosomes on FMR1 mRNA caused by ASO-CCG binding (Fig. 2e) may increase its half-life, an observation that was previously reported for hundreds of mRNAs ${ }^{30,31}$.

FMR1 transcripts containing $\mathrm{CGG}^{\exp }$ have been found in nuclear inclusions both in the brains of patients with FXTAS and in cell models 9,13 . Many proteins required for RNA maturation, transport, stability, and translation are efficiently sequestered by binding directly or indirectly to $\mathrm{rCGG}^{\exp 10-16}$. We showed that ASO-CCG led to a general increase in miRNA levels and corrected aberrant splicing events in cells overexpressing CGG ${ }^{\exp }$, which suggests that ASO-CCG released DGCR8 and SAM68 from sequestration (Fig. 1e, f and Supplementary Fig. S1f). The activity of other proteins affected by binding to rCGG ${ }^{\text {exp }}$ may also be restored by ASO-CCG. Previously, we applied a similar strategy in a mouse model of myotonic dystrophy, in which ASO blockers targeting $\mathrm{CUG}^{\mathrm{exp}}$ corrected the alternative splicing of hundreds of genes due to the release of MBNLs (muscleblindlike splicing regulators) from pathogenic sequestration on

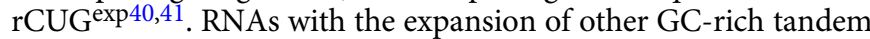
repeats, such as (rGGGGCC)n or (rCUG)n, were reported to form gel-like nuclear inclusions in a repeat length-dependent manner ${ }^{42,43}$. These processes can enhance the sequestration efficiency of RBPs. Therefore, abolishing the interaction of such proteins with $\mathrm{rCGG}^{\exp }$ and also the interactions between different rCGG ${ }^{\exp }$ molecules should globally reduce RNAmediated toxicity.

The toxicity of FMRpolyG was proven in Drosophila and mouse models ${ }^{18,19,44}$. Mice expressing the full-length $5^{\prime} \mathrm{UTR}$ of

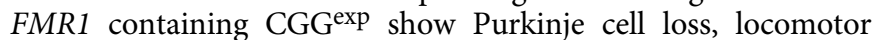
impairment and decreased viability, while mice in which the sequence controlling RAN translation initiation located upstream of CGG ${ }^{\exp }$ was deleted do not exhibit such symptoms ${ }^{19}$. Recently, the expression of rCGG ${ }^{\text {exp }}$ was shown to inhibit global translation and to activate the cellular stress response ${ }^{45}$. In turn, stressinduced phosphorylation of eIF $2 \alpha$ enhances RAN translation ${ }^{45}$. These data suggest that RAN translation is part of a feed-forward loop that may lead to neurodegeneration ${ }^{45}$. As ASO-CCG decreases the level of toxic FMRpolyG production (Figs. 2b, c, $4 \mathrm{f}$ and Supplementary Fig. S2a-c), it may significantly slow disease progression. Moreover, the results of our transcriptome-based studies showed that the levels of genes responsible for translation were rescued in ASO-CCG-treated FXTAS mice (Fig. 5a). The decrease in FMRpolyG biosynthesis from mutant FMR1 mRNA may occur at different stages of translation, e.g., initiation or elongation. By binding individually to $\mathrm{rCGG}^{\mathrm{exp}}$, ASO-CCGs can prevent either the formation of the initiation complex during the scanning phase of the $43 \mathrm{~S}$ ribosome subunit or the stalling and dissociation of the $80 \mathrm{~S}$ ribosome. Our sucrose-gradient fractionation experiments, which showed an increase in the level of $\mathrm{CGG}^{\text {exp }}$-carrying mRNAs in the free mRNA and monoribosome fractions after ASO-CCG treatment suggest that ASO-CCG can block the translation of both FMRP and FMRpolyG (Fig. 2e). A similar strategy for suppressing the translation of toxic proteins was applied in cell models of Huntington disease and spinocerebellar ataxia type 3 (SCA3), neurodegenerative disorders caused by the dominant effect of trinucleotide repeat expansions ${ }^{46}$. Targeting the CAG repeats with ASOs in a cellular model 
selectively decreased the biosynthesis of proteins from mutant mRNA carrying expanded CAG repeats in an open reading frame but not of normal huntingtin and ataxin- $3^{46}$. Moreover, ASO targeting the initiation site of rCGG $^{\exp }$ RAN translation in FMR1 mRNA was able to reduce the level of toxic FMRpolyG and at the same time increase FMRP in cell models ${ }^{24}$. Our data show that ASO-CCG reduces the level of FMRpolyG and the number of FMRpolyG foci in cell models and in vivo (Figs. 2b, c, 4f and Supplementary Fig. S2a-c), but it may also reduce the steady-state level of FMRP (Fig. 3e and Supplementary Fig. 2c). This observation should be carefully considered when planning potential FXTAS therapies as induction of FMRP deficiency in adults may lead to impairment of neuronal functions ${ }^{47}$. Thus, determining the appropriate dose of ASO-CCG to ensure both the preservation of sufficient FMRP levels and a beneficial reduction in the pathogenic processes causing FXTAS should be the goal of future studies.

In the P90CGG DOX-induced mouse model, the transgene containing human $5^{\prime} \mathrm{UTR}$ of FMR1 with CGG ${ }^{\exp }$ outside of the context of murine Fmr1 is controlled by a prion gene promoter, with the highest expression in the hippocampus, striatum, and cerebellum, particularly in lobule $\mathrm{X}$. This phenotype is accompanied by the greatest number of intranuclear FMRpolyG inclusions in the above-mentioned brain regions and by behavioral impairments connected with their functions $s^{32,33}$. The time course of phenotype induction in this model has been carefully established. After 12 weeks of DOX induction, mice exhibited a phenotype of decreased motor performance, while after 8 weeks of exposure, most mice remained asymptomatic ${ }^{33}$. No spontaneous recovery of motor functions and no decrease in the number of inclusions in cerebellar lobule X could be observed once these phenotypes have developed ${ }^{33}$, demonstrating the need for early intervention. On the other hand, the stability of the phenotype even after cessation of the 12-week transgene induction, as well as the absence of an overt toxicity phenotype or increased mortality in these mice, provide an ideal framework for testing the in vivo effectiveness of the ASO treatment. Therefore, we adopted a $4+$ $4+4$ schedule (Fig. 4b) with constant ASO-CCG infusion over 4 weeks starting at week 4 of DOX administration (for a total of 12 weeks of DOX induction). Thereby, it was possible to reduce the formation of FMRpolyG inclusions in cerebellar lobule X along with significant improvement of motor performance compared to saline-treated controls (Fig. 4d-f). However, it is not clear at this point whether the improvement in motor performance is the direct result of the reduction in the number or size of inclusions in lobule $\mathrm{X}$ or a correlate. On the other hand, this reduction is a strong indication of the potency of the ASO-CCG because lobule $\mathrm{X}$ is a region, where the transgene expression is particularly high in our P90CGG model. An interesting question to be addressed is whether this intervention is sufficient to provide lasting protection or merely slows down the pathogenic processes. Based on the encouraging findings of our current study we believe that further preclinical assessment of ASO-CCG that takes into consideration potential long-lasting effects of different treatment protocols, as well as the influence of age and treatment onset, is warranted in P90CGG mutants and other FXTAS models.

Cerebellar lobule $\mathrm{X}$ has been widely used to monitor inclusion formation in the P90CGG model. However, it should be considered that other cerebellar regions as well as basal ganglia also show high transgene expression and are involved in motor performance and disease pathology. Therefore, to address further functional changes, we have investigated the therapeutic potential of ASO-CCG at the transcriptome level from striatum tissue. In ASO-CCG-treated mice, the expression levels of FXTAS-related genes were similar to the expression levels observed in control animals (Fig. 5a). One example is the group of genes responsible for the regulation of translation and mitochondrial function. In FXTAS cell models these processes are impaired ${ }^{45,48}$. Treatment with ASO-CCG also increased the level of mRNAs carrying short CGG repeats located mostly in the $5^{\prime} \mathrm{UTR}$, but this effect was statistically significant for only $\sim 14 \%$ of such transcripts (Fig. 5b). However, upon ASO-CCG treatment, the level of protein products of selected genes was not affected in mouse brains and slightly reduced in human cells for only two such genes (Fig. $5 \mathrm{c}$ and Supplementary Fig. S6c, d). Another adverse effect of ASO-CCG observed at the mRNA level was the induction of a few genes controlling the immune response (Fig. $5 \mathrm{~d}$ and Supplementary Table S1). Nevertheless, the induction of these genes did not result in detectable changes at the steady-state protein levels in the ASO-CCG treated brains (Fig. 5e and Supplementary Fig. S6e).

In summary, we showed that short oligonucleotide steric blockers composed exclusively of LNA units targeting rCGGexp can affect multiple stages of mutant FMR1 gene expression. ASO-CCG can interfere with cotranscriptional R-loop formation and DNA damage, RNA-mediated toxicity, and toxic protein biosynthesis, indicating its compelling therapeutic potential for the treatment of FXTAS, FXPOI, and other diseases caused by CGG $^{\exp }{ }^{49}$. The potency of ASO-CCG was clearly manifested in the amelioration of behavioral and molecular phenotypes in the FXTAS mouse model. Importantly, the therapeutic strategy presented in this work, which relies on targeting RNA containing expanded microsatellite repeats with ASO steric blockers, could also be used to treat other diseases with similar etiology via cerebrospinal fluid delivery.

\section{Methods}

Antisense oligonucleotides. The 9 or 11-nucleotide-long ASOs were composed of 8 or 10 LNA units, respectively, and a $2^{\prime}-\mathrm{O}-\mathrm{Me}$ unit at $3^{\prime}$ end. All LNA positions were phosphorothioated. The sequence of ASO-CCGs is $5^{\prime}$-CCGCCGCCGCC- $3^{\prime}$ (11 nucleotides long) or 5'-CCGCCGCCG-3' (9 nucleotides long), the sequence of ASO-ctrl is 5'-TTGAACATAAG-3' (11 nucleotides long) or 5'-TGAACATAA-3' (9 nucleotides long) and ASO-CCG-Cy3 is 9-nucleotide-long oligonucleotide $5^{\prime}$ CCGCCGCCG- $3^{\prime}$ with Cy3 modification at a $5^{\prime}$ end. Toxic ASO used as a positive control in viability and apoptosis assays is 15-nucleotide-long ASO composed of LNA units (bolded) located at $5^{\prime}$ and $3^{\prime}$ ends and DNA nucleotides in the central core. All positions were phosphorothioated. The sequence of toxic ASO is $5^{\prime}$-CCGCCGCCGCCGCCG-3'. ASOs were synthesized and HPLC purified by Kaneka Eurogentec.

Genetic constructs. Genetic constructs containing CGG repeats used in this work were previously described: $(20 \times \mathrm{CGG}, 60 \times \mathrm{CGG})^{13},(100 \times \mathrm{CGG})^{19}$ and $(100 \times \mathrm{CGG}-$ mCherry $)^{23}$. The $20 \times C G G$ and $60 \times$ CGG contain bare CGG repeats without flanking sequences of FMR1 gene. The $100 \times$ CGG contains $5^{\prime}$ UTR sequence of FMR1 gene with 100 CGG repeats and located downstream GFP sequence and the product of its overexpression is fusion FMRpolyG-GPF protein. The 100×CGG-mCherry contains 5'UTR sequence of FMR1 gene with 100 CGG repeats and located downstream mCherry sequence and products of its expression are FMRpolyG and mCherry which translation starts from FMRP-specific start codon (FMRP-N-mCherry). MB1-40-mCherry construct ${ }^{50}$ was used in experiments based on the detection of fluorescent proteins after SDS-PAGE separation (FL-SDS-PAGE method, referred to as reference mCherry construct). MBNL3-39GFP construct ${ }^{50}$ was used in flow cytometry (referred as control GFP construct). SMN2 minigene (pCI SMN2) was a gift from Elliot Androphy (Addgene plasmid \# 72287; http://n2t.net/addgene:72287; RRID:Addgene_72287) ${ }^{51}$. The SAM68independent control, Atp2a1 minigene, was described in ref. ${ }^{52}$.

Electrophoretic mobility shift assay (EMSA). The $\mathrm{rCGG}_{20}$ was a gift from W. Krzyżosiak (The Institute of Bioorganic Chemistry, Polish Academy of Sciences). $5^{\prime}$ radiolabeling of $\mathrm{rCGG}_{20}$ was performed as previously described ${ }^{52}$ followed by 1 -min denaturation step. EMSA was carried out by incubating $5^{\prime}$ radiolabeled $\mathrm{rCGG}_{20}(1 \mathrm{nM})$ with 9 or 11 -nucleotide-long ASO-CCG of indicated concentrations (ranging from 0 to $500 \mathrm{nM}$ ). Reactions were performed in a volume of $10 \mu \mathrm{l}$, in $1 \times$ buffer $\mathrm{E}(50 \mathrm{mM} \mathrm{NaCl}, 50 \mathrm{mM} \mathrm{KCl}, 50 \mathrm{mM}$ TRIS- $\mathrm{HCl}, 1 \mathrm{mM} \mathrm{MgCl}$, pH 8.0) and incubated at $37^{\circ} \mathrm{C}$ for $15 \mathrm{~min}$. The samples were run on a native $8 \% \mathrm{PA}$ gel in $0.5 \times \mathrm{TBE}$ at $100 \mathrm{~V}$ for $1 \mathrm{~h}$. The gel was subsequently dried, and the signal was detected overnight, visualized on IP through FLA-1500 (FujiFilm) and quantified 
using Multi Gauge 3.0 software (FujiFilm). $K_{d}$ measurement of the $\mathrm{rCGG}_{20} /$ ASO-CCG interaction was based on the percentage of free $\mathrm{rCGG}_{20}$ using one phase decay curve in GraphPad program, represented by an equation $\mathrm{Y}=(\mathrm{Y} 0-$ Plateau $)^{*} \exp \left(-\mathrm{K}^{*} \mathrm{X}\right)+$ Plateau.

Cell culture and transfection. COS7 cells were grown in a high glucose DMEM medium with L-glutamine (Lonza), 10\% fetal bovine serum (Merck), $1 \%$ antibiotic/ antimycotic (Merck) at $37^{\circ} \mathrm{C}$ in $5 \% \mathrm{CO}_{2}$. Fibroblasts (CGG ${ }^{\text {norm}} /-$ (2), $\mathrm{C} 0603$;

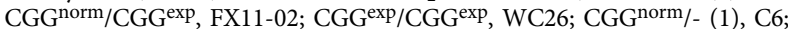
CGG $\exp /-$, F3; CGGnorm/- (3), GM23963; CGGnorm/- (4), GM04033) were previously described ${ }^{53,54}$ and grown in EMEM medium (Lonza), 15\% fetal bovine serum (Merck), 1\% MEM nonessential amino acids (Thermo Fisher Scientific) and $1 \%$ antibiotic/antimycotic (Merck) at $37^{\circ} \mathrm{C}$ in $5 \% \mathrm{CO}_{2}$. For transfection with genetic constructs, COS7 cells were plated on the appropriate cell culture vessels. Cells were transfected $2 \mathrm{~h}$ from plating with genetic constructs at $\sim 80 \%$ confluency. After 3-4 h, cells were transfected with ASOs. Fibroblasts were plated on appropriate cell culture vessels and transfected at $\sim 80 \%$ confluency. In all experiments, ASOs were denatured before transfection for $30 \mathrm{~s}$ at $95^{\circ} \mathrm{C}$ and chilled on ice. All transfections were made with the use of Lipofectamine 3000 (Thermo Fisher Scientific) according to the manufacturer's instructions. Specific conditions for all experiments are stated below.

Monitoring of cell viability in real time. For cell viability assay, COS7 cells were plated on a 96-well plate in $150 \mu \mathrm{l}$ of media. The next day, cells were transfected with 9-nucleotide-long ASOs and toxic ASO at $200 \mathrm{nM}$ concentration. After $6 \mathrm{~h}$ transfection mixture was removed and replaced with $150 \mu \mathrm{l}$ of media containing real-time cell viability reagents (Real-Time-Glo MT Cell Viability Assay, Promega). The luminescence signal was read on a Tecan Spark reader set after 7, 14, 23, 32, 46 , and $64 \mathrm{~h}$. At the time of transfection cell, confluency was about $40 \%$ and reached $90 \%$ in mock control after $52 \mathrm{~h}$ of culture.

Apoptosis assay. For apoptosis assay, COS7 cells were plated in a 24-well plate and transfected $5 \mathrm{~h}$ from plating with 9-nucleotide-long ASOs and toxic ASO at $200 \mathrm{nM}$ concentration. As additional positive control cells treated with $50 \mu \mathrm{M}$ CCCP (carbonyl cyanide 3-chlorophenylhydrazone). After $24 \mathrm{~h}$, apoptosis of the cells was tested with Guava MitoDamage Kit (Luminex) according to the manufacturer's protocol. Samples were analyzed on a guava easyCyte HT flow cytometer and guavaSoft" software, version 3.1.1 (Luminex) using parameters and gating strategy assigned to dedicated MitoDamage Kit software template. Cells with lower MitoSense Red fluorescence were considered as positive for apoptosis (Supplementary Fig. S7).

miRNA-level quantification. For mature miRNA-level quantification after treatment with ASOs, we used COS7 cells, 12 -well plates, $1 \mu \mathrm{g}$ of $100 \times$ CGG construct per well, and 9-nucleotide-long ASOs at $200 \mathrm{nM}$ concentration. For mature miRNA level quantification upon expression of normal or expanded CGG repeats, we used COS7 cells, 24 -well plate, and 500 ng of $20 \times$ CGG or $60 \times$ CGG or $100 \times$ CGG construct per well. After $48 \mathrm{~h}$ or $72 \mathrm{~h}$, cells were harvested using TRI Reagent (Merck) and the total RNA was isolated according to the manufacturer's instructions or with the use of Total RNA Zol-Out ${ }^{\mathrm{TM}} \mathrm{D}$ (A\&A Biotechnology). RNA quantity and quality were checked with the use of a spectrophotometer (DeNovix). Complementary DNA (cDNA) was synthesized in a coupled polyadenylation reverse-transcription reaction by using $2 \mu \mathrm{g}$ of the total RNA for $1 \mathrm{~h}$ at $37^{\circ} \mathrm{C}$ in RT buffer (10 mM Tris- $\mathrm{HCl} \mathrm{pH} \mathrm{8.0,75} \mathrm{mM} \mathrm{KCl,} 10 \mathrm{mM} \mathrm{DTT}, 70 \mathrm{mM}$ $\mathrm{MgCl}_{2}, 20 \mathrm{U}$ RNasin, $2.5 \mathrm{mM}$ of all four deoxynucleoside triphosphates, $0.5 \mathrm{mM}$ of rATP, and $800 \mathrm{ng}$ of anchored oligo(dT) primer with a sequence $5^{\prime}$-GTG CAG GGT CCG AGG TTC AAC TAT AGG TTT TTT TTT TTT TTT TTT TTT TTT VN-3' supplemented with $200 \mathrm{U}$ of Superscript III reverse transcriptase (Thermo Fisher Scientific) and $5 \mathrm{U}$ of E. coli poly(A) polymerase (PAP; New England Biolabs). Reactions were heat-inactivated for $10 \mathrm{~min}$ at $85^{\circ} \mathrm{C}$. Then, $2 \mu$ lof the $9 x$ diluted cDNA template was used for each $\mathrm{qPCR}$ with a universal reverse primer (UR) with sequence identical to the part of anchored oligo(dT) primer (Supplementary Table S1) and a probe-specific forward primer $(600 \mathrm{nM}$ each). qRT-PCR was performed using the Power SYBR Green PCR Master Mix (Thermo Fisher Scientific), and samples were run in technical triplicates on a 7900HT Fast RealTime PCR instrument. Ct values were normalized against the internal controls, $U 6$. Fold differences in expression level were calculated according to the $2^{-\Delta \Delta \mathrm{Ct}}$ method. All primers are listed in Supplementary Table S2.

Alternative splicing analysis. For SAM68-dependent alternative splicing analysis upon CGG ${ }^{\exp }$ expression, we used COS7 cells, 24-well plate, $300 \mathrm{ng}$ of either $20 \times \mathrm{GG}$ or $60 \times \mathrm{CGG}$ or $100 \times \mathrm{CGG}$ construct, $100 \mathrm{ng}$ of $S M N 2$ minigene and $100 \mathrm{ng}$ of Atp2a1 minigene per well and 11-nucleotide-long ASOs at 100 or $200 \mathrm{nM}$ concentration. After $48 \mathrm{~h}$, cells were harvested using TRI Reagent (Merck), and the total RNA was isolated according to the manufacturer's instructions. cDNA was synthesized with the use of GoScript ${ }^{\mathrm{TM}}$ Reverse Transcriptase System (Promega) and random primers (Promega) according to the manufacturer's instructions. PCR was performed with GoTaq DNA Polymerase and primers listed in Supplementary Table S2. Sequences of SMN2_F and SMN2_R primers were obtained from ${ }^{13}$. PCR products were separated in 1-2\% agarose gel with ethidium bromide. Images were captured with the use of G:Box EF2 (Syngene). Signals of PCR products were assessed with the use of GeneTools (Syngene). Percent of alternative exon inclusion (PSI, the percent of alternative exon spliced in) was calculated based on signals of two bands according to the following formula (isoform with included exon*100)/ (isoforms with included exon + excluded exon).

Analysis of ASO-CCG cellular uptake. COS7 cells were plated on a 96-well plate for epifluorescence microscopy or on $\mu$-Slide 8 Well (Ibidi) for confocal microscopy. ASO-CCG-Cy3 was introduced to cells via transfection or added directly to the cell at $200 \mathrm{nM} 3-4 \mathrm{~h}$ after plating. For epifluorescence microscopy after $24 \mathrm{~h}$ cell medium was replaced with PBS. Images were taken with Axio Observer.Z1 microscope equipped with AxioCam MRm camera, filter set excitation 540-552, emission 575-640, A-Plan $\times 10 / 0.25$ Ph1 objective (Zeiss), and AxioVs40 module. For confocal microscopy, Hoechst 33342 (Thermo Fisher Scientific) was added to cell medium to final concentration $5 \mu \mathrm{g} / \mathrm{ml} 24 \mathrm{~h}$ post ASO delivery, and cells were incubated for $15 \mathrm{~min}$ at $37^{\circ} \mathrm{C}$. Images were captured with the use of a Nikon A1Rsi confocal microscope with Nikon Apo $\times 40$ WI $\lambda$ S DIC N2 objective. Hoechst 33342 and $\mathrm{Cy} 3$ were excited with $405 \mathrm{~nm}$ diode laser and $561 \mathrm{~nm}$ pumped-diode laser, respectively. For detection dichroic mirror 405/488/561 nm with spectral filters, $450 / 50 \mathrm{~nm}$ and $595 / 50 \mathrm{~nm}$ were used.

Flow cytometry. For assessment of total FMRpolyG-GFP, we used COS7 cells, 48-well plate $250 \mathrm{ng}$ of $100 \times \mathrm{CGG}$ or control GFP per well, and 11-nucleotide-long ASOs at 100 or $200 \mathrm{nM}$. After $48 \mathrm{~h}$, cells were analyzed with the use of flow cytometry protocol described in detail in ref. ${ }^{23}$. Shortly, cells were trypsinized and suspended in warm PBS. Propidium iodide (PI) at final concentration $1 \mu \mathrm{g} / \mathrm{ml}$ was added to stain dead cells which were next excluded from analysis. GFP and PI signal in cells was analyzed with guava easyCyteTM HT flow cytometer and guavaSoft $^{\text {tw }}$ software, version 3.1.1 Luminex). The gating strategy is presented in Supplementary Fig. S8.

\section{Microscopic analysis of FMRpolyG-GFP inclusions. For assessment of} FMRpolyG-GFP inclusions, we used COS7, 96-well plate, $125 \mathrm{ng}$ of $100 \times \mathrm{CGG}$ construct per well and 11-nucleotide-long ASOs at 100 or $200 \mathrm{nM}$ concentration. After $48 \mathrm{~h}$, the culture medium was removed and cells were washed in warm PBS to remove dead cells. Next, cells were incubated in a cell culture medium with Hoechst 33342 (Thermo Fisher Scientific) at a final concentration $5 \mu \mathrm{g} / \mathrm{ml}$ at $37^{\circ} \mathrm{C}$ for $30 \mathrm{~min}$ prior analysis. Images were taken with Axio Observer.Z1 microscope equipped with AxioCam MRm camera, filter set excitation 450-490, emission 515-565 (GFP) and filter set excitation 335-383, emission 420-470 (Hoechst 33342), A-Plan $\times 10 / 0.25 \mathrm{Ph} 1$ objective (Zeiss), and AxioVs40 module. A number of nuclei and inclusions were calculated with Image $1.51 \mathrm{j} 8$ and $3 \mathrm{~d}$ object counter plugin $^{55}$.

Quantification of CGG containing mRNAs in COS7 cells. For assessment of the level of mRNAs containing CGG repeats, transfection of COS7 with 100×CGG construct and ASOs and total mRNA isolation was performed as described in "miRNA level quantification". Next, cDNA was synthesized with the use of GoScript ${ }^{\mathrm{TM}}$ Reverse Transcriptase System (Promega) according to the manufacturer's instructions with the exception of the first-strand synthesis reaction temperature which was $37^{\circ} \mathrm{C}$. We used an anchored oligo(dT) primer containing oligo dT tract which allowed for exclusive reverse transcription of polyA+ RNAs. qPCR was performed with the use of iTaq ${ }^{\text {Tw }}$ Universal SYBR ${ }^{\oplus}$ Green Supermix (BioRad) according to manufacturer's instructions and analyzed with the use of QuantStudio 7 Flex Real-Time PCR System machine (Thermo Fisher Scientific). To ensure amplification of 100×CGG construct mRNA but not DNA following primers were used: forward primer complementary to the $3^{\prime}$ part of GFP sequence and universal reverse (UR) primer. Primers for amplification of endogenous mRNA of FMR1 and NUB1 were set in $3^{\prime} \mathrm{UTR}$ of transcripts. Ct values were normalized against GAPDH and amplified with gene-specific forward primer set in $3^{\prime}$ part of mRNA and UR primer. All primers are listed in Supplementary Table S2. Fold differences in expression level were calculated according to the $2^{-\Delta \Delta \mathrm{Ct}}$ method.

Sucrose-gradient fractionation. For assessment of mRNAs association with ribosomes, we used COS7 cells at 30-40\% confluency, T75 bottles $\left(75 \mathrm{~cm}^{2}\right), 20 \mu \mathrm{g}$ of 100xCGG construct per bottle, and 9-nucleotide-long ASOs at $200 \mathrm{nM}$ concentration. 3.5-4 mln of cells were used to prepare the single sample. After $48 \mathrm{~h}$, cells were incubated for $30 \mathrm{~min}$ in cell medium with cycloheximide (Merck) at a final concentration $100 \mu \mathrm{g} / \mathrm{ml}$. Cells were washed with PBS, trypsinized, centrifuged, suspended in lysis buffer $(20 \mathrm{mM}$ Tris- $\mathrm{HCl} \mathrm{pH} 7.5,10 \mathrm{mM} \mathrm{NaCl}, 3 \mathrm{mM}$ $\mathrm{MgCl}_{2}, 1 \mathrm{mM}$ DTT, $0.3 \%$ Triton X-100, $50 \mathrm{mM}$ sucrose, $1 \mathrm{mM}$ RNasin (Promega), $100 \mu \mathrm{g} / \mathrm{ml}$ cycloheximide), and vortexed. Lysates were centrifuged at $10,000 \times g$ for $10 \mathrm{~min}$. Supernatants were transferred to new probes and proper volumes of $1 \mathrm{M}$ $\mathrm{NaCl}$ and $1 \mathrm{M} \mathrm{MgCl}_{2}$ were added to adjust final concentrations to $170 \mathrm{mM}$ and $13 \mathrm{mM}$, respectively. Linear $15-45 \%$ sucrose gradient was prepared in a buffer (25 mM Tris-HCl pH 7.5, $25 \mathrm{mM} \mathrm{NaCl}, 5 \mathrm{mM} \mathrm{MgCl}, 100 \mu \mathrm{g} / \mathrm{ml}$ cycloheximide) in Open-Top Polyclear Tubes (tube size: 9/16" X 3 1/2", Seton Scientific) with the use of Biocomp Gradient Station (BioComp Instruments) and incubated for $30 \mathrm{~min}$ at 
$4^{\circ} \mathrm{C}$. Lysates were carefully loaded on gradients and ultracentrifuged at $160,000 \times g$ at $4{ }^{\circ} \mathrm{C}$ for $2 \mathrm{~h} 40 \mathrm{~min}$ in SW41 Ti swing-out rotor and Optima L100XPN Ultracentrifuge (Beckman Coulter). Next, 12 fractions of $0.97 \mathrm{ml}$ were collected with the use of Piston Gradient Fractionator (BioComp Instruments) and 2110 Fraction Collector (Biorad) at a $0.3 \mathrm{~mm} / \mathrm{s}$ speed, with a distance of $6.75 \mathrm{~mm}$ per fraction. Absorbance was monitored at $254 \mathrm{~nm}$ (A254) with an EM-1 Econo UV monitor (Biorad). Fractions were frozen at $-80^{\circ} \mathrm{C}$ and then used for total RNA isolation. $0.5 \mu \mathrm{l}$ of GlycoBlue ${ }^{\mathrm{mt}}$ Coprecipitant $(15 \mathrm{mg} / \mathrm{ml}$, Thermo Fisher Scientific) was added to fractions. $5 \mathrm{M} \mathrm{NaCl}$ was added to obtain a final concentration of $0.1 \mathrm{M}$. Equal volume of isopropanol $(\sim 1 \mathrm{ml})$ was added, mixed, and incubated for $30 \mathrm{~min}$ at $-20^{\circ} \mathrm{C}$. Samples were centrifuged at $12,000 \times g$ for $10 \mathrm{~min}$ at $4{ }^{\circ} \mathrm{C}$. Pellet was resuspended in $50 \mu \mathrm{l}$ of ultrapure water and vortexed with $0.5 \mathrm{ml}$ of TriReagent (Merck). Next, the total RNA was isolated according to the manufacturer's instructions with the addition of $1 \mu \mathrm{l}$ of GlycoBlue $\mathrm{e}^{\mathrm{ry}}$ Coprecipitant $(15 \mathrm{mg} / \mathrm{ml}$, Thermo Fisher Scientific) and sodium acetate $3 \mathrm{M} \mathrm{pH} 5.5$ (Thermo Fisher Scientific) to a final concentration of $0.3 \mathrm{M}$ to the aqueous phase. RNA was diluted in $15 \mu$ of ultrapure water. In total, $5.6 \mu$ of RNA solution was used for cDNA synthesis which was performed as described under "Quantification of CGG containing mRNAs in COS7 cells". PCR and gel electrophoresis were performed as described under "Alternative splicing analysis", with primers listed in Supplementary Table S2. qPCR was performed as described under "Quantification of CGG containing mRNAs in COS7 cells".

\section{Quantification of FMR1 mRNA and pre-mRNA upon RNase H1 insufficiency.} For quantification of the effect of RNase H1 insufficiency and ASOs on FMR mRNA and pre-mRNA level, fibroblasts were seeded on a 12-well plate and transfected at $\sim 80 \%$ of confluency with siRNAs (future synthesis) at a final concentration of $15 \mathrm{nM}$. siRNA sequences are listed in Supplementary Table S2. After $24 \mathrm{~h}$, the 11-nucleotide long (CGG $\left.{ }^{\text {norm} /-~(2), ~} \mathrm{CGG}^{\text {norm} / C G G}{ }^{\exp }, \mathrm{CGG}^{\exp } / \mathrm{CGG}^{\exp }\right)$ or 9-nucleotide long (CGGnorm/- (1)) ASOs at $200 \mathrm{nM}$ final concentration were delivered. Fibroblasts were harvested $48 \mathrm{~h}$ post ASOs delivery. The isolation of RNA was performed with the Total RNA Zol-Out ${ }^{\text {tw }}$ D kit (A\&A Biotechnology). In total, 300-500 ng of the total RNA was used for reverse transcription with GoScript ${ }^{\mathrm{TM}}$ Reverse Transcriptase (Promega) and random primers (Promega). qPCR was performed with the use of iTaq Tn $^{\text {"I }}$ Universal SYBR ${ }^{\Phi}$ Green Supermix (BioRad) according to the manufacturer's instructions and analyzed with the use of QuantStudio 7 Flex Real-Time PCR System machine (Thermo Fisher Scientific) with primers listed in Supplementary Table S2. Ct values were normalized against $G A P D H$. Fold differences in expression level were calculated according to the $2^{-\Delta \Delta \mathrm{Ct}}$ method

In vitro transcription. In vitro transcription reactions presented in the main figures were performed on the $100 \times$ CGG construct linearized by Avr II which digest the genetic construct at the end of the 5'UTR sequence. The construct contains T7 polymerase promoter upstream to $5^{\prime} \mathrm{UTR}$ of $F M R 1$. Reactions were performed with $\sim 500 \mathrm{ng}$ of template DNA in $10 \mu \mathrm{l}$ of a mixture containing $1 \times$ transcription buffer (Promega), $10 \mathrm{mM}$ DTT, $10 \mathrm{U}$ of RNasin (Promega), rNTPs ( $0.5 \mathrm{mM}$ each), $4 \mathrm{U}$ of T7 polymerase (Promega) and (if required) $2.5 \mu \mathrm{M}$ 9-nucleotide-long ASO-ctrl, ASO-CCG or ASO-CCG-Cy3. In the case of RNase H treatment, 1 U of RNase H (Thermo Fisher Scientific) was added to the mixture of proper samples at the beginning of the reaction. Samples without RNase $\mathrm{H}$ were treated with an equal volume of $50 \%$ glycerol to provide the same density of the mixture. The reactions were performed at $37^{\circ} \mathrm{C}$ for either $20 \mathrm{~min}$ (the full saturation of transcript by ASO during the whole reaction) or $90 \mathrm{~min}$ (the full saturation of transcript only at the beginning of the reaction). Transcription reactions were stopped by the addition of $6 \times$ BLUE DNA Loading buffer (Blirt) which contains $60 \mathrm{mM}$ EDTA. All in vitro transcription reaction products were analyzed on $1 \%$ agarose gels with or without ethidium bromide $(0.5 \mu \mathrm{g} / \mathrm{ml})$ run in $1 \mathrm{x}$ Tris-Borate-EDTA buffer at $70 \mathrm{~V}$ for $2 \mathrm{~h}$. To visualize the nucleic acid products, the agarose gels were scanned using Amersham Typhoon RGB Biomolecular Imager. Nucleic acids with intercalated ethidium bromide and the signal coming from ASO-CCG-Cy3 were detected using Cy3 filter. Intensities of the transcript signals were measured and quantitated with Multi Gauge 3.0 software (Fujifilm) and ImageQuant TL 8.1.0.0 (Cytiva). Each signal (gels stained with $\mathrm{EtBr}$ ) was normalized upon the intensity of the signal coming from a genetic construct in the same lane. This procedure was applied to compensate for random variations in the sample signal intensities due to gel loading errors. Note that the first R-loop visualization was tested by two approaches: (1) In vitro transcription performed on the non-digested 100xCGG construct (Supplementary Fig. S3b, left panel), and (2) In vitro transcription performed on the 100xCGG construct digested with Avr II (Supplementary Fig.3b, right panel). In vitro transcription confirming R-loops formation was performed on the digested 100xCGG construct (Supplementary Fig. S3c). Detailed procedures are provided in the Supplementary Figures' description.

Cytoplasm/nucleus fractionation. For cell fractionation of the nuclei and cytoplasm, fibroblasts were seeded on a 100 -mm plate and transfected at $\sim 80 \%$ of confluency with 9-nucleotide-long ASOs at $200 \mathrm{nM}$ final concentration. Fibroblasts were harvested $48 \mathrm{~h}$ post ASOs delivery, washed in ice-cold $1 \times$ PBS and centrifuged at $500 \times g$ for $5 \mathrm{~min}$. Cell pellets were resuspended by gentle pipetting in $1 \mathrm{ml}$ ice-cold HLB buffer (hypotonic lysis buffer: $10 \mathrm{mM}$ Tris- $\mathrm{HCl}$ (pH 7.5), $10 \mathrm{mM}$ $\mathrm{NaCl}, 3 \mathrm{mM} \mathrm{MgCl}, 0.3 \%$ (v/v) NP-40, 10\% (v/v) glycerol), complemented with RNasin (Promega). Mix was incubated on ice $30 \mathrm{~min}$ and vortexed briefly. Cells were centrifuged at $2000 \times g$ for $5 \mathrm{~min}$ at $4^{\circ} \mathrm{C}$. The supernatant (cytoplasmic fraction) was transferred to a new tube, kept on ice, and $5 \mathrm{M} \mathrm{NaCl}$ solution was added to adjust the $\mathrm{NaCl}$ concentration to $140 \mathrm{mM}$. The nuclei pellet was washed $4 \mathrm{x}$ by adding HLB, pipetting, and centrifuging at $500 \times g$ for $2 \mathrm{~min}$ at $4{ }^{\circ} \mathrm{C}$. The total RNA was isolated with the use of TRI Reagent (Merck) according to the manufacturer's instructions. cDNA synthesis and qPCR were performed as described under "Quantification of FMR1 mRNA and pre-mRNA upon RNase H1 insufficiency" with primers listed in Supplementary Table S2.

Western blot. For analysis of proteins level CGGnorm/- (2), CGG $\mathrm{exp}^{\mathrm{e}} \mathrm{CGG}^{\exp }$ fibroblasts were seeded on a $100-\mathrm{mm}$ plate and transfected at $~ 80 \%$ of confluency with 11-nucloetide long ASOs at $200 \mathrm{nM}$ final concentration. Fibroblasts were harvested $48 \mathrm{~h}$ post ASO delivery. Cell extracts were prepared in lysis buffer $(\mathrm{pH} 8$ $150 \mathrm{mM} \mathrm{NaCl}, 1 \% \mathrm{NP}-40,1 \times$ protease inhibitor (Roche) and $1 \mathrm{mM}$ PMSF), vortexed, and frozen at $-80^{\circ} \mathrm{C}$. Protein extracts were centrifuged at $11,300 \times g$ for 10 min at $4{ }^{\circ} \mathrm{C}$, measured by Pierce ${ }^{\mathrm{ms}} \mathrm{BCA}$ Protein Assay Kit (Thermo Fisher Scientific), and heat-denatured for $5 \mathrm{~min}$ at $95^{\circ} \mathrm{C}$ with the addition of standard sample buffer. Electrophoresis and wet transfer were performed with the use of Mini-PROTEAN Tetra System (Bio-Rad). In all, $30-40 \mu \mathrm{g}$ of protein per well was separated in $10 \%$ SDS polyacrylamide gel in Laemmli buffer. Proteins were transferred to the nitrocellulose membrane (GE Healthcare) for $1 \mathrm{~h}, 100 \mathrm{~V}$ in Laemmli buffer with $20 \%$ methanol. Membranes were blocked in $5 \%$ nonfat dry milk in PBS with $0.1 \%$ Tween 20 (PBS-T) overnight at $4{ }^{\circ} \mathrm{C}$. Incubation with antibodies was performed in following conditions: rabbit anti-FMRP antibody (ab17722, Abcam) 1:1000, rabbit anti-KANSL1L antibody (NBP2-14139, Novus Biologicals) 1:125, rabbit anti-DAZAP1 (PA5-41887, Thermo Fisher Scientific) 1:2000 in 5\% nonfat dry milk in PBS-T for $4.5 \mathrm{~h}$ at $4{ }^{\circ} \mathrm{C}$, mouse anti-GAPDH antibody (sc-47724, Santa Cruz Biotechnology) 1:8000/1:10,000 for $1 \mathrm{~h}$ at RT. Membranes were washed in PBS-T and incubated with horseradish peroxidaseconjugated secondary antibodies, anti-rabbit (AS09 602, Agrisera) 1:2500 in PBS-T for $1 \mathrm{~h}$ at RT or anti-mouse (sc-2005, Santa Cruz Biotechnology) 1:20,000 (antiGAPDH) in PBS-T for $45 \mathrm{~min}$ at RT. Membranes were washed in PBS-T and covered with SuperSignal ${ }^{\text {Dx }}$ West Femto Maximum Sensitivity Substrate (Thermo Fisher Scientific) according to the manufacturer's instructions. Images were captured with the use of G:Box Chemi-XR5 (Syngene) and analyzed with the use of GeneTools 4.02 (Syngene). For analysis of proteins level CGGnorm/- (1), $\mathrm{CGG}^{\text {norm} /-~(3), ~} \mathrm{CGG}^{\text {norm} /-~(4), ~} \mathrm{CGG}^{\exp } /$ - fibroblasts were seeded on $100 \mathrm{~mm}$ plate or 6-well plates and transfected at $\sim 80 \%$ of confluency with 9-nucloetide long ASOs at $200 \mathrm{nM}$ final concentration. Fibroblasts were harvested $48 \mathrm{~h}$ post ASO delivery. Cell extracts were prepared in lysis buffer ( $\mathrm{pH} 8,150 \mathrm{mM} \mathrm{NaCl}, 1 \% \mathrm{NP}$ $40,1 \times$ protease inhibitor (Roche) and $1 \mathrm{mM}$ PMSF), vortexed, and frozen at $-80^{\circ} \mathrm{C}$. Next, protein cellular extracts were centrifuged at $11,300 \times g$ for $10 \mathrm{~min}$ at $4{ }^{\circ} \mathrm{C}$, measured by Pierce ${ }^{m m}$ BCA Protein Assay Kit (Thermo Fisher Scientific), and heat-denatured for $10 \mathrm{~min}$ at $70^{\circ} \mathrm{C}$ with the addition of Bolt LDS buffer (Invitrogen). In total, 20-30 $\mu \mathrm{g}$ of protein per well was separated in Bolt ${ }^{\mathrm{mi}} 4-12 \%$ BisTris Plus gel (Invitrogen) in Bolt ${ }^{\mathrm{m}}$ MES SDS Running Buffer. Proteins were transferred to PVDF transfer membrane (GE Healthcare) for $1 \mathrm{~h}, 100 \mathrm{~V}$ in Leammli buffer with $20 \%$ methanol. Western blot experiments were performed using SNAP ID Protein Detection System (Merck Millipore). Membranes were blocked with $0.125 \%$ nonfat dry milk in TBS with $0.1 \%$ Tween 20 (TBS-T) for $20 \mathrm{~min}$. Incubation with antibodies was performed in following conditions: rabbit anti-FMRP antibody (ab17722, Abcam) 1:500 in $0.125 \%$ nonfat dry milk in TBS-T for $1 \mathrm{~h} 10 \mathrm{~min}$, mouse anti-GAPDH antibody (sc-47724, Santa Cruz Biotechnology) 1:10,000 for 15 min, rabbit anti-SLC40A1 antibody (NBP1-21502, Novus Biologicals) 1: 1000 for $1 \mathrm{~h}$, rabbit anti-QKI antibody (ab126742, Abcam) 1:1000 for $1 \mathrm{~h}$, rabbit anti-VKORC1L1 antibody (PA5-48618, Thermo Fisher) 1:300 for $1 \mathrm{~h}$, rabbit anti-alpha-tubulin antibody (ab52866, Abcam) 1:10,000 for $15 \mathrm{~min}$. Membranes were washed in TBS-T and incubated with horseradish peroxidaseconjugated secondary antibodies-anti-rabbit (A9169, Merck) 1:20,000 for 15 min or anti-mouse (A9044, Merck) 1:20,000 and washed with TBS. Antibody-antigen complexes were visualized by enhanced chemiluminescence (ECL) using Luminata Forte HRP Substrate (Merck) and detected with G:BoxSystem (Syngene). For analysis of protein level in mice hippocampus tissues, samples were lysed with the help of a dounce homogenizer (Wheaton) in denaturing lysis buffer (1\% sodium dodecyl sulfate, $1 \mathrm{mM}$ EDTA, $20 \mathrm{mM}$ Tris- $\mathrm{HCl}, 1$ tablet Pierce protease inhibitor (Thermo Fisher Scientific)). Samples were homogenized by agitation for $5 \mathrm{~min}$ at $95^{\circ} \mathrm{C}$. After centrifugation for $1 \mathrm{~min}$ at $16,000 \times g$, the supernatant was collected for further processing. The protein concentration of each sample was determined using the Bradford assay (Bio-Rad) according to the manufacturer's instructions. In all, $20 \mu \mathrm{g}$ protein from each sample was separated by SDS-PAGE (12\%) and transferred to PVDF membranes (Merck). Membranes were first blocked in Intercept Blocking Buffer (Li-COR), then incubated overnight at $4{ }^{\circ} \mathrm{C}$ with primary antibodies: rabbit anti-GFAP (NB300-141, Novus Biologicals) 1:5000, rabbit antiTNF-alpha (ab66579, Abcam) 1:750, rabbit anti-CD68 (ab125212, Abcam) 1:500, mouse anti-alpha-tubulin (T6199, Merck) 1:10,000 and antigen-antibody complexes were visualized via incubation with fluorescent secondary antibodies: antirabbit IRDye 800CW (926-32211, Li-COR) 1:15000 and anti-mouse IRDye 680RD 
(926-68070, Li-COR) 1:15000 for $1 \mathrm{~h}$ at RT. Florescent blotted membranes were scanned with the Odyssey scanner (LI-COR) and the signal associated with the antigen/antibody complexes were analyzed using FIJI-ImageJ software. For analysis of protein level in mice cortex tissues, samples were placed in tubes with $50 \mu \mathrm{l}$ of lysis buffer ( $\mathrm{pH} 8,150 \mathrm{mM} \mathrm{NaCl}, 1 \% \mathrm{NP}-40,1 \times$ protease inhibitor (Roche), and $1 \mathrm{mM}$ PMSF), homogenized with the use of Omni Tissue Homogenizer (Omni International), incubated for $30 \mathrm{~min}$ on ice, vortexed and frozen at $-80{ }^{\circ} \mathrm{C}$ overnight. Then samples were processed as samples derived from CGG

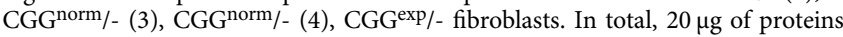
were separated. For detection of FMRP, QKI, VKORC1L1, and SLC40A1 procedures were the same as for fibroblasts samples. For detection of AIF1 and PPP1R1B, membranes were blocked with $1 \%$ bovine serum albumin (BSA) in TBS$\mathrm{T}$ for $20 \mathrm{~min}$. Incubation with antibodies was performed in following conditions: rabbit anti-alpha tubulin antibody (ab52866, Abcam) 1:10,000 in 1\% BSA in TBS-T for 15 min, rabbit anti-AIF1 antibody (019-19741, Wako) 1:500 for $1 \mathrm{~h}$, rat antiPPP1R1B antibody (MAB4230, R\&D Systems) 1:500 for $1 \mathrm{~h}$. Membranes were washed in TBS-T with $0,5 \mathrm{M} \mathrm{NaCl}$ and incubated with horseradish peroxidaseconjugated secondary antibody anti-rabbit (A9169, Merck) 1:20,000 for 15 min or rhodamine-conjugated (TRITC) anti-rat (712-025-153, JIR) 1:10,000 for $15 \mathrm{~min}$. Membranes were washed with TBS with $0.5 \mathrm{M} \mathrm{NaCl}$ and antibody-antigen complexes were visualized Luminata Forte HRP Substrate (Merck) and G:BoxSystem (Syngene) for anti-rabbit and by Amersham Typhoon RGB Biomolecular Imager with $\mathrm{Cy} 5$ filter for anti-rat antibody. Intensities of the protein signals were measured and quantitated with ImageQuant TL 8.1.0.0 (Cytiva). For detection of the signal from different antibodies, the membrane was cropped or washed with stripping buffer (1.5\% glycine, $0.1 \%$ SDS, $1 \%$ Tween $20, \mathrm{pH} 2.2$ ).

Animals. All experimental procedures were approved by local ethics committee Landesverwaltungsamt Sachsen-Anhalt (CEEA\# 42502-2-1219UniMD) and met the guidelines of local and European regulations (European Union directive no. 2010/ 63/EU). Inducible bitransgenic animals (referred to as P90CGG throughout the text) were obtained by crossing single transgenic mutants carrying a 90CGG repeat tract along with a tetracycline responsive element (TRE-90CGG) with the transgenic prion protein-reverse tetracycline transactivator (PrP-rtTA) driver line, as described previously ${ }^{32}$. Mice were weaned at 4 weeks of age and group-housed in standard laboratory cages containing standard bedding material and Nestlets $\mathrm{s}^{\mathrm{mm}}$ nesting material (Ancare). Animals were kept in temperature and humidity-controlled conditions with an inverted 12-h light/12-h dark cycle. Water and food were provided ad libitum. Genotyping was performed from tail biopsies that were incubated overnight at $55^{\circ} \mathrm{C}$ in DirectPCR tail lysis reagent (Viagen) and $0.3 \mathrm{mg} / \mathrm{ml}$ proteinase $\mathrm{K}$ (Carl Roth). Following heat inactivation, $1 \mu \mathrm{l}$ from each sample was used for the genotyping PCR, performed according to the previously described protocol ${ }^{56}$.

Doxycycline (DOX) administration. P90CGG mice were provided $4 \mathrm{~g} / \mathrm{kg}$ DOX containing food pellets (ssniff) for a period of 12 weeks starting from the day of weaning. At the end of the 12-week of exposure, DOX-containing food pellets were replaced with standard mouse feed until sacrifice. The same DOX induction schedule with P90CGG mice has previously shown to result in motor deficits and a high load of intranuclear inclusions in the cerebellum ${ }^{33}$.

Stereotactic surgery and ASO administration. At weaning, P90CGG were randomly allocated to one of the two treatment groups (saline versus ASO-CCG) with animals from the same litter being represented in both treatment groups. 8-week old mice were anesthetized in an isoflurane anesthesia system (Rothacher) with 5\% isoflurane in $\mathrm{O}_{2} / \mathrm{N}_{2} \mathrm{O}$ mixture and placed on a stereotactic frame (World Precision Instruments). Anesthesia was maintained throughout the surgery at $1.5-2.0 \%$. A $28 \mathrm{G}$, brain infusion cannula (Plastics1) with 2.5 -mm cut length that is attached via a catheter to a primed osmotic pump (Alzet model: 1004; Durect) filled with $100 \mu \mathrm{l}$ of 11-nucleotide-long ASO solution $(\sim 5.88 \mu \mathrm{g} / \mu \mathrm{l})$ was inserted at coordinates $\mathrm{M} / \mathrm{L}$ : -0.11 (directed laterally to the right), A/P: -0.05 relative to bregma resulting in an intracerebroventricular infusion at the right lateral ventricle. The cannula was secured to its location with Paladur ${ }^{\oplus}$ dental cement (Kulzer). Pumps were subcutaneously implanted on the back of the mice posterior to the scapulae. Osmotic pumps were removed under anesthesia at the end of the 4-week infusion schedule. Control animals were infused with $0.9 \%$ saline solution. Cannula placement was visually validated for each animal during cryosectioning.

Motor behavior. At the end of the 12-week DOX induction period, P90CGG mice were subjected to a 3-day motor performance test on Rotarod equipment (Ugo Basile). On day one, mice were trained at $15 \mathrm{rpm}$ constant speed on the rotating rod for a maximum of $60 \mathrm{~s}$ in four trials. On the second day, animals were tested for each of the five different speeds $(15,24,31,36,40 \mathrm{rpm})$ in two trials for a maximum of $60 \mathrm{~s}$. The third day consisted of testing the mice during a 5-min accelerated ramp from 4 to $40 \mathrm{rpm}$ in four trials. In each trial, the latency to fall off the rod was registered.

Tissue processing. Animals were anesthetized in an isoflurane chamber, sacrificed by decapitation, and then brains were immediately removed. Right hemispheres are drop fixed overnight at $4{ }^{\circ} \mathrm{C}$ in $4 \%$ paraformaldehyde/PBS and cryoprotected in
$30 \%$ sucrose/PBS containing $0.02 \%$ sodium azide. Fixed brain samples were then embedded in Tissue-Tek ${ }^{\circ}$ O.C.T. ${ }^{m}$ compound (Sakura) and frozen in liquid nitrogen-cooled isopentane. Frozen blocks were cut in $7 \mu \mathrm{m}$ thick sagittal sections in a freezing microtome (Leica). Sections were mounted on poly-L-lysine coated slides, air-dried at room temperature, and stored at $4{ }^{\circ} \mathrm{C}$ for fluorescent immunohistochemistry. For RNA-seq, the striatum and the cortex (excluding the prefrontal cortex) of the mice have been dissected out from the left hemispheres in icecold ACSF and immediately snap-frozen in liquid nitrogen. The samples were kept at $-80^{\circ} \mathrm{C}$ until further processing.

FMRpolyG immunohistochemistry. Slide mounted sections were treated with $0.01 \mathrm{M}$ sodium citrate $(\mathrm{pH}=6.0)$ in a microwave oven for antigen retrieval. An extra antigen retrieval step with proteinase $\mathrm{K}$. $(5 \mu \mathrm{g} / \mathrm{ml})$ was included to better visualize the inclusions. Sections were first blocked for endogenous biotin and then blocked for mouse Ig using M.O.M. reagents (Vectorlabs). Sections were incubated overnight at $4{ }^{\circ} \mathrm{C}$ in mouse-FMRpolyG $\left(8 \mathrm{FM}^{57} ; 1: 200\right)$ antibody. Following biotinylated secondary antibody incubation (mouse on mouse immunodetection kit, BMK-2202, Vector Labs), antigen-antibody complexes were visualized by incubation in Cy5-tagged streptavidin (Invitrogen). Sections were counterstained with DAPI and coverslipped with Immu-Mount ${ }^{\mathrm{m}}$ (Shandon). The granular layer of the cerebellum lobule $\mathrm{X}$ was selected for this quantification. Fluorescent photomicrographs of cerebellum lobule X with a $\mathrm{z}$-step size of $2 \mu \mathrm{m}$ were registered under an epifluorescence microscope (Leica) at 630x magnification and analyzed with open-source image processing software Fiji 2.0.0-rc-69/1.52p. 400 DAPI + nuclei that lie within the granular layer of the lobule $\mathrm{X}$ were counted per mice by a researcher who was blind to the treatment groups. For this quantification, random images of $30 \mu \mathrm{m}$ by $30 \mu \mathrm{m}$ generated from whole photomicrographs of lobule $\mathrm{X}$ by a custom cell counter script developed for Fiji software were used. For each DAPI + nuclei that were counted, the script recorded through the researcher's input whether or not it contains a FMRpolyG + intranuclear inclusion and its longitudinal size. From the data of the identified inclusions, a percentage and an average size value were calculated for each mouse. Statistics have been performed from these values.

RNA-seq and bioinformatics analysis. Frozen cortex or striatum samples were placed in tubes with $600 \mu \mathrm{l}$ of TriReagent (Merck) and homogenized with the use of Omni Tissue Homogenizer (Omni International). Total RNA was isolated with the use of Direct-zol ${ }^{\text {st }}$ RNA MiniPrep kit (Zymo Research) according to the manufacturer's instructions with DNase I treatment. RNA quantity was checked with the use of Qubit ${ }^{\circ}$ RNA BR Assay Kit and Qubit Fluorometer (Thermo Fisher Scientific) according to manufacturer's instructions, RNA quality was checked with the use of RNA 6000 Nano Kit and 2100 Bioanalyzer System (Agilent) according to the manufacturer's instructions. Library preparation and total RNA sequencing ( $2 \times 100 \mathrm{bp})$ were performed by CeGaT (Germany) with the use of $100 \mathrm{ng}$ of RNA, TruSeq Stranded Total RNA kit, and NovaSeq 6000. Demultiplexing of the sequencing reads was performed with Illumina bcl2fastq (2.19). Adapters were trimmed with Skewer (version 0.2.2) ${ }^{58}$. Reference Mus musculus (GRCm38, primary assembly) genome and annotations were downloaded from Ensembl (ver. 91 or 93). Quality and adapter trimming of short reads was performed using Trimmomatic $0.39^{59}$. Short reads matching known rRNA sequences were removed using HISAT2 2.1.0 aligner ${ }^{60}$. Read quality reports before and after quality filtering were prepared using FastQC 0.11.5 software (http://www.bioinformatics.babraham. ac.uk/projects/fastqc/). Filtered reads were aligned to the reference genomes using STAR 2.7.1a algorithm ${ }^{61}$. Read mapping reports were created using Qualimap 2.2.2 software ${ }^{62}$. RSEM 1.3.0 (RNA-Seq by Expectation Maximization ${ }^{63}$ ) was used to quantify the expression values of genes. Hierarchical clustering of RNA-seq samples (Pearson correlation metric, centroid linkage) based on the expression values of all genes was performed using standard R 3.3.1 functions (R Core Team 2016) and variance stabilizing transformation provided by DESeq 2 package ${ }^{64}$. Differential expression analysis between designated groups of samples was performed using voom+limma ${ }^{65}$ pipeline with settings described in ${ }^{66}$. The edgeR 3.14.0 package was used for initial data filtering and normalization using the TMM method $^{66}$. Voom function from the limma 3.28 .21 package ${ }^{66}$ was employed to estimate the mean-variance relationship in the data. Afterward, linear modeling and empirical Bayes moderation were applied to identify differentially expressed genes between groups of interest as described in ref. ${ }^{66} . P$ value was generated using moderated $t$-statistic and adjusted for multiple testing using Benjamini-Hochberg's method (adjusted $p$-value, adj.P.Val). adj.P.Val threshold of 0.01 and fold change threshold of 1.1 were used during the analysis. RNA-seq data were stored in NCBI SRA database. SRA accession: PRJNA577423.

Gene ontology analysis. For gene ontology analysis, data from differential expression analysis of striatum or cortex RNA-seq results were used. List of genes expressed on arbitrarily determined level (AveExpr $>1$; AveExpr -average expression across all samples, in $\log 2 \mathrm{CPM}$ ) and significantly upregulated or downregulated (Adj.P.Val $\leq$ 0.05; Adj.P.Val - Benjamini-Hochberg false discovery rate adjusted $P$ value) was extracted for saline vs control and ASO-CCG vs control groups. A reference list of genes was prepared from all genes detected in striatum or cortex tissue which were expressed on an arbitrarily determined level (AveExpr $>1$ ). 
The analysis was performed with the use of PANTHER14.1 program. The analysis type was PANTHER Overrepresentation Test, annotation version and the release date was GO Ontology database released 2019-02-02. Annotation datasets were GO biological process complete, GO molecular function complete, and Reactome pathways. Test type was Fisher's exact with the use of Bonferroni correction for multiple testing.

FL-SDS-PAGE. For assessment of soluble FMRpolyG-GFP after ASO-CCG treatment, we used COS7 cells, 48 -well plate, $100 \mathrm{ng}$ of $100 \times$ CGG construct, $100 \mathrm{ng}$ of $100 \times$ CGG-mCherry construct, and $50 \mathrm{ng}$ of reference mCherry construct per well and 11-nucleotide-long ASOs at 125 or $200 \mathrm{nM}$. After $48 \mathrm{~h}$ cells were harvested and proceeded according to FL-SDS-PAGE protocol described with details in ref. ${ }^{23}$. Shortly, cells were lysed, vortexed, and sonicated. In total, $10 \mu$ l of lysate without previous heat denaturation was electrophoretically separated in $12 \%$ polyacrylamide SDS gel. The fluorescence signal was captured directly in the gel with the use of Amersham Typhoon RGB Biomolecular Imager.

\section{Quantification of mRNAs and pre-mRNAs containing CGG and CCG repeats.} For analysis of mRNAs and pre-mRNAs level fibroblasts were seeded on 100-mm plate and transfected at $\sim 80 \%$ of confluency with 11-nucleotide-long ( $\mathrm{CGG}^{\text {norm} /-}$ (2), $\mathrm{CGG}^{\text {norm}} / \mathrm{CGG}^{\exp }, \mathrm{CGG}^{\mathrm{exp}} / \mathrm{CGG}^{\exp }$ ) or 9-nucleotide long (CGG ${ }^{\text {norm} /-~(1), ~}$ $\mathrm{CGG}^{\exp /-)}$ ASOs at $200 \mathrm{nM}$ final concentration. Fibroblasts were harvested $48 \mathrm{~h}$ post ASOs delivery. RNA isolation, cDNA synthesis and qPCR were performed as described under "Cytoplasm/nucleus fractionation" with primers listed in Supplementary Table S2. PCR and gel electrophoresis were performed as described under "Alternative splicing analysis" with primers listed in Supplementary Table S2.

\section{Assessment of efficiency of ASOs delivery via gymnosis using flow cyto-}

metry. Assessment of efficiency of ASOs delivery via gymnosis was performed as described under "Flow cytometry". 9-nucleotide-long ASOs (ASO-CCG and ASOctrl) were delivered via transfection at $200 \mathrm{nM}$ concentration or directly to the culture medium (gymnosis) at 200 or $400 \mathrm{nM}$ concentration (ASO-CCG).

\section{Quantification of the expression level of TRE-nCGG-eGFP transgene and} immune system-related markers in P90CGG mouse. The expression levels of TRE-nCGG-eGFP transgene and immune system-related markers upon ASO-CCG delivery were quantified from total RNA isolated from striatal and cortical tissue, respectively, of saline or ASO-CCG treated mice. cDNA synthesis and $\mathrm{qPCR}$ were performed as described under "Quantification of FMR1 mRNA and pre-mRNA upon RNase H1 insufficiency" with primers listed in Supplementary Table S2.

Heatmap. Heatmap was created based on differential expression analysis result from the striatum of control and P90CGG mouse. Only genes with Adj.P.Val $<0.05$ were selected for further analysis. The set contained 417 genes from the saline vs control group. In the final results, one gene "ENSMUSG00000050121_Opalin" was removed because of its outlier characteristics. Samples marked with numbers in the range 1-4 belonged to the saline group, samples marked with numbers in the range 5-8 belonged to the ASO-CCG group, and samples marked with numbers in the range 9-12 belonged to the control group. Genes present in twelve samples were visualized using a three-color heatmap. Data for all samples were organized in order from left to right in 12 columns plus the first column describing a particular gene. For every gene (row) and every sample in a given gene, a Z-score was calculated using the average and standard deviation of four control samples. Resulting Z-scores for every sample in every row (gene) were saved in tabdelimited text files with a header and then converted into the heatmap. Heatmap was generated using R "pheatmap" library version 1.0.12. Both rows (genes) and samples (columns) were clustered using complete-linkage method (clustering_method = "complete" option in pheatmap package). Z-scores for every gene (rows) and samples (columns) are represented with 50 shades between orange, white, and green. Results are centered so that Z-score equal to zero is represented by white.

Statistics and reproducibility. All data obtained in this study were processed and analyzed with the use of Microsoft Excel. Statistical analysis of all experiments performed on cell lines as well as western blots performed on mice tissues was done using unpaired two-tailed Student's $t$ test. The normality of the data has been verified using Lilliefors test. Tests that result in $P<0.05$ have been reported to be statistically significant. The symbols; ${ }^{*}, * *, * * *$ represent values of $P<0.05, P<0.01$, $P<0.001$, respectively. All data were analyzed using Statistica software version 10 (TIBCO Software) and Prism software version 7 (GraphPad). Error bars represent standard deviation (SD). Statistical analysis on the behavior data was done using two-way repeated-measures ANOVA for training and constant speed rotarod, and unpaired two-tailed Student's $t$ test for accelerating rotarod test. The FMRpolyG+ nuclei counts and size were analyzed using unpaired two-tailed Student's $t$ test. The normality of the data has been verified using Shapiro-Wilk test whenever $t$-statistics have been used to demonstrate statistical significance. Tests that result in
$P<0.05$ have been reported to be statistically significant. The symbols; $*, * *, * * *$ represent values of $P<0.05, P<0.01, P<0.001$, respectively. All data were analyzed using Prism software version 7 (GraphPad). Error bars represent SEM for rotarod tests and SD for the FMRpolyG+ nuclei counts and size. Pearson correlation and Mann-Whitney test applied to RNA-seq data analysis were calculated using Prism software version 7 (GraphPad). All cellular and in vitro experiments presented in this work was repeated at least two times with similar results.

Reporting summary. Further information on research design is available in the Nature Research Reporting Summary linked to this article.

\section{Data availability}

RNA-seq data generated and analyzed during this study are available in the NCBI SRA database. SRA accession: PRINA577423. Publicly available datasets from https://www. ensembl.org/ were used in this study. The code used to generate the heatmap presented in Supplementary Fig. S5 is available in the GitHub repository, https://github.com/ MagdalenaDerbis/R-script-to-generate-heatmap/blob/main/Supplementary\%20R\% 20 script $\% 20$ to\%20generate\%20heatmap.R. Other data that support the findings of this study are available from the corresponding author upon reasonable request. Source data are provided with this paper.

Received: 23 December 2019; Accepted: 24 December 2020 Published online: 24 February 2021

\section{References}

1. Hagerman, R. J. et al. Fragile X syndrome. Nat. Rev. Dis. Prim. 3, 17065 (2017)

2. Hagerman, R. J. et al. Intention tremor, parkinsonism, and generalized brain atrophy in male carriers of fragile X. Neurology 57, 127-130 (2001).

3. Hagerman, R. J. \& Hagerman, P. Fragile X-associated tremor/ataxia syndrome-features, mechanisms and management. Nat. Rev. Neurol. 12, 403-412 (2016)

4. Glineburg, M. R., Todd, P. K., Charlet-Berguerand, N. \& Sellier, C. Repeatassociated non-AUG (RAN) translation and other molecular mechanisms in Fragile X Tremor Ataxia syndrome. Brain Res. 1693, 43-54 (2018).

5. Tassone, F. et al. Elevated levels of FMR1 mRNA carrier males: a new mechanism of involvement in the fragile-X syndrome. Am. J. Hum. Genet. 66 , 6-15 (2000).

6. Loomis, E. W., Sanz, L. A., Chédin, F. \& Hagerman, P. J. Transcriptionassociated R-loop formation across the human FMR1 CGG-repeat region. PLoS Genet. 10, e1004294 (2014).

7. Groh, M., Lufino, M. M. P., Wade-Martins, R. \& Gromak, N. R-loops associated with triplet repeat expansions promote gene silencing in Friedreich ataxia and fragile X syndrome. PLoS Genet. 10, e1004318 (2014)

8. Diab, M. A. et al. The g-rich repeats in FMR1 and C9orf72 loci are hotspots for local unpairing of DNA. Genetics 210, 1239-1252 (2018)

9. Tassone, F., Iwahashi, C. \& Hagerman, P. J. FMR1 RNA within the intranuclear inclusions of fragile $\mathrm{X}$-associated tremor/ataxia syndrome (FXTAS). RNA Biol. 1, 103-105 (2004).

10. Iwahashi, C. K. et al. Protein composition of the intranuclear inclusions of FXTAS. Brain 129, 256-271 (2006)

11. Sofola, O. A. et al. RNA-binding proteins hnRNP A2/B1 and CUGBP1 suppress fragile X CGG premutation repeat-induced neurodegeneration in a Drosophila model of FXTAS. Neuron 55, 565-571 (2007).

12. Jin, P. et al. Pur $a$ binds to rCGG repeats and modulates repeat-mediated neurodegeneration in a Drosophila model of fragile X tremor/ataxia syndrome. Neuron 55, 556-564 (2007).

13. Sellier, C. et al. Sam 68 sequestration and partial loss of function are associated with splicing alterations in FXTAS patients. EMBO J. 29, 1248-1261 (2010).

14. Qurashi, A., Li, W., Zhou, J. Y., Peng, J. \& Jin, P. Nuclear accumulation of stress response mRNAs contributes to the neurodegeneration caused by fragile X premutation rCGG repeats. PLoS Genet. 7, e1002102 (2011).

15. Sellier, C. et al. Sequestration of DROSHA and DGCR8 by expanded CGG RNA repeats Alters microRNA processing in fragile X-associated tremor/ ataxia syndrome. Cell Rep. 3, 869-880 (2013).

16. Cid-Samper, F. et al. An integrative study of protein-RNA condensates identifies scaffolding RNAs and reveals players in fragile X-associated tremor/ ataxia syndrome. Cell Rep. 25, 3422-3434.e7 (2018).

17. Banez-Coronel, M. \& Ranum, L. P. W. Repeat-associated non-AUG (RAN) translation: insights from pathology. Lab. Investig. 99, 929-942 (2019).

18. Todd, P. K. et al. CGG repeat-associated translation mediates neurodegeneration in fragile X tremor ataxia syndrome. Neuron $\mathbf{7 8}, 440-455$ (2013). 
19. Sellier, C. et al. Translation of expanded CGG repeats into FMRpolyG is pathogenic and may contribute to fragile $\mathrm{X}$ tremor ataxia syndrome. Neuron 93, 331-347 (2017).

20. Disney, M. D. et al. A small molecule that targets $r(C G G){ }^{\exp }$ and improves defects in fragile X-associated tremor ataxia syndrome. ACS Chem. Biol. 7, 1711-1718 (2012).

21. Tran, T. et al. Targeting the r(CGG) repeats that cause FXTAS with modularly assembled small molecules and oligonucleotides. ACS Chem. Biol. 9, 904-912 (2014).

22. Yang, W. Y. et al. Small molecule recognition and tools to study modulation of $\mathrm{r}(\mathrm{CGG}) \exp$ in fragile $\mathrm{X}$-associated tremor ataxia syndrome. ACS Chem. Biol. 11, 2456-2465 (2016).

23. Derbis, M., Konieczny, P., Walczak, A., Sekrecki, M. \& Sobczak, K. Quantitative evaluation of toxic polyglycine biosynthesis and aggregation in cell models expressing expanded CGG repeats. Front. Genet. 9, 216 (2018).

24. Rodriguez, C. M. et al. A native function for RAN translation and CGG repeats in regulating fragile X protein synthesis. Nat. Neurosci. 23, 386-397 (2020).

25. Sobczak, K. et al. Structural diversity of triplet repeat RNAs. J. Biol. Chem. 285, 12755-12764 (2010)

26. Napierala, M., Michalowski, D., de Mezer, M. \& Krzyzosiak, W. J. W. J. Facile FMR1 mRNA structure regulation by interruptions in CGG repeats. Nucleic Acids Res. 33, 451-463 (2005).

27. Studzińska, S., Cywoniuk, P. \& Sobczak, K. Application of ion pair chromatography coupled with mass spectrometry to assess antisense oligonucleotides concentrations in living cells. Analyst 144, 622-633 (2019).

28. Kozlowski, P., de Mezer, M. \& Krzyzosiak, W. J. Trinucleotide repeats in human genome and exome. Nucleic Acids Res. 38, 4027-4039 (2010).

29. Crossley, M. P., Bocek, M. \& Cimprich, K. A. R-loops as cellular regulators and genomic threats. Mol. Cell 73, 398-411 (2019).

30. Mauger, D. M. et al. mRNA structure regulates protein expression through changes in functional half-life. Proc. Natl Acad. Sci. USA 116, 24075-24083 (2019).

31. $\mathrm{Wu}, \mathrm{Q}$. et al. Translation affects mRNA stability in a codon-dependent manner in human cells. eLife 8, e45396 (2019).

32. Hukema, R. K. et al. Reversibility of neuropathology and motor deficits in an inducible mouse model for FXTAS. Hum. Mol. Genet. 24, 4948-4957 (2015).

33. Castro, H. et al. Selective rescue of heightened anxiety but not gait ataxia in a premutation 90CGG mouse model of fragile X-associated tremor/ataxia syndrome. Hum. Mol. Genet. 26, 2133-2145 (2017).

34. Yang, Z. \& Wang, K. K. W. Glial fibrillary acidic protein: from intermediate filament assembly and gliosis to neurobiomarker. Trends Neurosci. 38, 364-374 (2015).

35. Schoch, K. M. \& Miller, T. M. Antisense oligonucleotides: translation from mouse models to human neurodegenerative diseases. Neuron 94, 1056-1070 (2017).

36. Mendell, J. R. et al. Eteplirsen for the treatment of Duchenne muscular dystrophy. Ann. Neurol. 74, 637-647 (2013).

37. Corey, D. R. Nusinersen an antisense oligonucleotide drug for spinal muscular atrophy. Nat. Neurosci. 20, 497-499 (2017).

38. Gebert, L. F. R. et al. Miravirsen (SPC3649) can inhibit the biogenesis of miR122. Nucleic Acids Res. 42, 609-621 (2014)

39. Obad, S. et al. Silencing of microRNA families by seed-targeting tiny LNAs. Nat. Genet. 43, 371-380 (2011).

40. Wheeler, T. M. et al. Reversal of RNA dominance by displacement of protein sequestered on triplet repeat RNA. Science 325, 336-339 (2009).

41. Wojtkowiak-Szlachcic, A. et al. Short antisense-locked nucleic acids (all-LNAs) correct alternative splicing abnormalities in myotonic dystrophy. Nucleic Acids Res. 43, 3318-3331 (2015).

42. Jain, A. \& Vale, R. D. RNA phase transitions in repeat expansion disorders. Nature 546, 243-247 (2017).

43. Fay, M. M., Anderson, P. J. \& Ivanov, P. ALS/FTD-associated C9ORF72 repeat RNA promotes phase transitions in vitro and in cells. Cell Rep. 21, 3573-3584 (2017).

44. Oh, S. Y. et al. RAN translation at CGG repeats induces ubiquitin proteasome system impairment in models of fragile $\mathrm{X}$-associated tremor ataxia syndrome. Hum. Mol. Genet. 24, 4317-4326 (2015).

45. Green, K. M. et al. RAN translation at C9orf72-associated repeat expansions is selectively enhanced by the integrated stress response. Nat. Commun. 8, 1-13 (2017).

46. $\mathrm{Hu}$, J. et al. Allele-specific silencing of mutant huntingtin and ataxin-3 genes by targeting expanded CAG repeats in mRNAs. Nat. Biotechnol. 27, 478-484 (2009).

47. Guo, W. et al. Ablation of Fmrp in adult neural stem cells disrupts hippocampus-dependent learning. Nat. Med. 17, 559-565 (2011).

48. Gohel, D. et al. FMRpolyG alters mitochondrial transcripts level and respiratory chain complex assembly in fragile $\mathrm{X}$ associated tremor/ataxia syndrome [FXTAS]. Biochim. Biophys. Acta Mol. basis Dis. 1865, 1379-1388 (2019).

49. Ishiura, $\mathrm{H}$. et al. Noncoding CGG repeat expansions in neuronal intranuclear inclusion disease, oculopharyngodistal myopathy and an overlapping disease. Nat. Genet. 51, 1222-1232 (2019).

50. Sznajder, L. J. et al. Mechanistic determinants of MBNL activity. Nucleic Acids Res. 44, 10326-10342 (2016).

51. Lorson, C. L., Hahnen, E., Androphy, E. J. \& Wirth, B. A single nucleotide in the SMN gene regulates splicing and is responsible for spinal muscular atrophy. Proc. Natl Acad. Sci. USA 96, 6307-6311 (1999).

52. Cywoniuk, P., Taylor, K., Sznajder, J. \& Sobczak, K. Hybrid splicing minigene and antisense oligonucleotides as efficient tools to determine functional protein/RNA interactions. Sci. Rep. 7, 17587 (2017).

53. Rovozzo, R. et al. CGG repeats in the 5'UTR of FMR1 RNA regulate translation of other RNAs localized in the same RNA granules. PLoS ONE 11, e0168204 (2016).

54. Garcia-Arocena, D. et al. Fibroblast phenotype in male carriers of FMR1 premutation alleles. Hum. Mol. Genet. 19, 299-312 (2010).

55. Bolte, S. \& Cordelières, F. P. A guided tour into subcellular colocalization analysis in light microscopy. J. Microsc. 224, 213-232 (2006).

56. Hukema, R. K. et al. Induced expression of expanded CGG RNA causes mitochondrial dysfunction in vivo. Cell Cycle 13, 2600-2608 (2014).

57. Buijsen, R. A. M. et al. FMRpolyG-positive inclusions in CNS and non-CNS organs of a fragile $\mathrm{X}$ premutation carrier with fragile $\mathrm{X}$-associated tremor/ ataxia syndrome. Acta Neuropathol. Commun. 2, 1-5 (2014).

58. Jiang, H., Lei, R., Ding, S.-W. \& Zhu, S. Skewer: a fast and accurate adapter trimmer for next-generation sequencing paired-end reads. BMC Bioinforma. 15, 182 (2014).

59. Bolger, A. M., Lohse, M. \& Usadel, B. Trimmomatic: a flexible trimmer for Illumina sequence data. Bioinformatics 30, 2114-2120 (2014).

60. Kim, D., Langmead, B. \& Salzberg, S. L. HISAT: a fast spliced aligner with low memory requirements. Nat. Methods 12, 357-360 (2015).

61. Dobin, A. et al. STAR: ultrafast universal RNA-seq aligner. Bioinformatics 29, 15-21 (2013)

62. Okonechnikov, K., Conesa, A. \& García-Alcalde, F. Qualimap 2: advanced multi-sample quality control for high-throughput sequencing data. Bioinformatics 32, 292-294 (2016)

63. Li, B. \& Dewey, C. N. RSEM: Accurate transcript quantification from RNASeq data with or without a reference genome. BMC Bioinformatics 12, 323 (2011).

64. Love, M. I., Huber, W. \& Anders, S. Moderated estimation of fold change and dispersion for RNA-seq data with DESeq2. Genome Biol. 15, 550 (2014).

65. Ritchie, M. E. et al. Limma powers differential expression analyses for RNAsequencing and microarray studies. Nucleic Acids Res. 43, e47 (2015).

66. Law, C. W. et al. RNA-seq analysis is easy as 1-2-3 with limma, Glimma and edgeR. F1000Research 5, 1408 (2016).

\section{Acknowledgements}

The authors wish to thank Nicolas Charlet-Berguerand for providing 8FM; antiFMRpolyG antibody and 20xCGG, 60xCGG, and 100xCGG genetic constructs, Anita Bhattacharyya for providing C0603, FX11-02, and WC26 fibroblasts and Paul J. Hagerman for providing C6 and F3 fibroblasts. This work was supported by the National Centre for Research and Development grant ERA-NET-E-Rare- 2/III/DRUG_FXSPREMUT/01/2016 (to K.S.), by the Ministry of Science and Higher Education of the Republic of Poland, from the quality-promoting subsidy, under the Leading National Research Centre (KNOW) program for the years 2012-2017 [KNOW RNA Research Centre in Poznan (No. 01/KNOW2/2014)], by the Foundation for Polish Science, TEAM POIR.04.04.00-00-5C0C/17-00 (to K.S.), by the Initiative of Excellence-Research University (05/IDUB/2019/94) at Adam Mickiewicz University, Poznan, Poland, and from the Poznan RNA Research Centre (to K.S.), by ERA-Net for Research Programmes on Rare Diseases grant 01GM1505 “Drug_FXSPreMut" (E-Rare-2 JTC 2014; to O.S.) and by the Deutsche Forschungsgemeinschaft (DFG, German Research Foundation) 362321501/RTG 2413 SynAGE to O.S.

\section{Author contributions}

K.S., O.S., and R.H. designed the study. M.D., E.K., D.N., M.S., A.P., and K.T. performed experiments and analyzed the data. E.K. performed all experiments on mouse models (Fig. 4c, d, e and Supplementary Fig. S4b), staining of FMRpolyG (Fig. 4f and Supplementary Fig. S4c), and western blots on hippocampus mouse tissues (Fig. 5e and Supplementary Figs. S6e, f). D.N. tested the effect of RNase H and ASOs on FMR1 transcript in fibroblast and in vitro (Fig. 3a-c and Supplementary Figs. S3a-c), assessed efficiency of ASOs delivery (Supplementary Fig. 4a), assessed level of CGG containing mRNAs (Supplementary Fig. S6c), and performed western blots on cortex mouse tissues and fibroblast cell lines (Figs. 3e, $5 \mathrm{c}$ and e and Supplementary Fig. S6d, e). M.S. performed cellular fractionation (Fig. 3d and Supplementary Fig. 3e), assessed level of FMR1 transcript and other CGG containing mRNAs (Supplementary Figs. S3d and S6c), 
quantified FMRP after ASO treatment (Fig. 3e), and level of mRNAs of immune systemrelated toxicity markers in mouse cortex (Fig. 5d). A.P. quantified level of miRNAs

(Fig. 1e and Supplementary Figs. S1c and d), assessed viability and apoptosis of cells after ASO treatment (Fig. 1c, d), and prepared Supplementary Table S1. K.T. assessed ASO affinity to CGG repeats in vitro (Fig. $1 \mathrm{~b}$ and Supplementary Fig. S1a). K.S. performed the analysis of gene expression changes in RNA-seq data (Fig. 5a, b and Supplementary Fig. S6b). M.D. performed all other experiments and prepared figures. M.D., E.K., and K.S. wrote the paper.

\section{Competing interests}

The authors declare no competing interests.

\section{Additional information}

Supplementary information The online version contains supplementary material available at https://doi.org/10.1038/s41467-021-21021-w.

Correspondence and requests for materials should be addressed to K.S.

Peer review information Nature Communications thanks the anonymous reviewer(s) for their contribution to the peer review of this work. Peer reviewer reports are available.
Reprints and permission information is available at http://www.nature.com/reprints

Publisher's note Springer Nature remains neutral with regard to jurisdictional claims in published maps and institutional affiliations.

\section{cc) (i)}

Open Access This article is licensed under a Creative Commons Attribution 4.0 International License, which permits use, sharing, adaptation, distribution and reproduction in any medium or format, as long as you give appropriate credit to the original author(s) and the source, provide a link to the Creative Commons license, and indicate if changes were made. The images or other third party material in this article are included in the article's Creative Commons license, unles indicated otherwise in a credit line to the material. If material is not included in the article's Creative Commons license and your intended use is not permitted by statutory regulation or exceeds the permitted use, you will need to obtain permission directly from the copyright holder. To view a copy of this license, visit http://creativecommons.org/ licenses/by/4.0/.

(C) The Author(s) 2021 